\title{
Nonadiabatic effects in the dissociation of oxygen molecules at the $\mathrm{Al}(111)$ surface
}

\author{
Jörg Behler,* Karsten Reuter, and Matthias Scheffler \\ Fritz-Haber-Institut der Max-Planck-Gesellschaft, Faradayweg 4-6, D-14195 Berlin, Germany \\ (Received 13 April 2007; revised manuscript received 15 February 2008; published 13 March 2008)
}

\begin{abstract}
The measured low initial sticking probability of oxygen molecules at the $\mathrm{Al}(111)$ surface that had puzzled the field for many years was recently explained in a nonadiabatic picture invoking spin-selection rules [J. Behler et al., Phys. Rev. Lett. 94, 036104 (2005)]. These selection rules tend to conserve the initial spin-triplet character of the free $\mathrm{O}_{2}$ molecule during the molecule's approach to the surface. A locally constrained densityfunctional theory approach gave access to the corresponding potential-energy surface (PES) seen by such an impinging spin-triplet molecule and indicated barriers to dissociation which reduce the sticking probability. Here, we further substantiate this nonadiabatic picture by providing a detailed account of the employed approach. Building on the previous work, we focus, in particular, on inaccuracies in present-day exchangecorrelation functionals. Our analysis shows that small quantitative differences in the spin-triplet constrained PES obtained with different gradient-corrected functionals have a noticeable effect on the lowest kinetic energy part of the resulting sticking curve.
\end{abstract}

DOI: 10.1103/PhysRevB.77.115421 PACS number(s): 82.20.Gk, 71.15.Mb, 68.49.Df, 34.20.-b

\section{INTRODUCTION}

The interaction of oxygen with metal surfaces plays a central role in many technologically relevant processes such as heterogeneous catalysis or corrosion. Key elementary steps in this interaction are the (dissociative) adsorption of the molecule at the surface, diffusion at or in the surface, and the (associative) desorption from the surface. Aiming to establish an atomic-scale understanding, detailed investigations study these interaction steps at well-defined model systems, often employing single crystal surfaces exposed to defined amounts of oxygen in an otherwise ultrahigh vacuum surrounding. A prominent example are studies of the dissociative adsorption of oxygen molecules at the $\mathrm{Al}(111)$ surface, which allegedly represents a most simple and basic case: the initial step in the oxygen interaction with a close-packed surface of a nearly free electron metal.

Surprisingly, even here such a fundamental issue such as the adsorption mechanism has not yet been settled. Several contradictory models such as the so-called "hot atom" motion, ${ }^{1,2}$ abstraction,,${ }^{3,4}$ or dissociation leading to neighboring adsorbed $\mathrm{O}$ atoms $\mathrm{s}^{5}$ have been proposed, mainly based on different interpretations of scanning tunneling microscopy data. Entangled with the mechanism is the sticking probability, which is defined as the ratio of sticking (dissociation) events to the total number of molecule-surface collisions. Many independent experiments have unambiguously shown that the initial sticking probability of thermal oxygen molecules at $\mathrm{Al}(111)$ is only about $1 \% .^{2,6-10}$ Furthermore, using molecular beam experiments, it was found that the sticking probability increases with translational kinetic energy of the impinging molecules and reaches a saturation value of about $90 \%$ only for kinetic energies higher than $0.5 \mathrm{eV} .{ }^{7}$ A straightforward explanation for this finding would be the existence of energy barriers toward dissociation that cannot be overcome by low energy, thermal molecules. Several firstprinciples theoretical studies employing density-functional theory (DFT) were carried out in order to identify these barriers on the potential-energy surface (PES), ${ }^{11-13}$ but were un- able to find any sizeable barriers. Consequently, although no explicit calculation of the sticking curve based on the highdimensional PES was done, it was concluded that the adiabatic PES studied by these DFT calculations cannot explain the experimental findings.

This has led to speculations that nonadiabatic effects may play an important role in the oxygen dissociation process at the $\mathrm{Al}(111)$ surface, ${ }^{12,14-19}$ just as much as for the interaction of $\mathrm{O}_{2}$ with several other metal surfaces. ${ }^{14,20-24}$ In an earlier letter, we investigated this role of nonadiabatic effects in the oxygen dissociation at $\mathrm{Al}(111)$ within a first-principles approach $^{25}$ and traced the nonadiabatic effects back to spinselection rules ${ }^{26}$ conserving the initial spin-triplet character of the free $\mathrm{O}_{2}$ molecule during the molecule's approach to the surface. In the present paper, we now give a detailed account of our approach and its underlying multifaceted methodology. This methodology comprises the calculation of spin-constrained PESs within our recently introduced locally constrained DFT (LC-DFT) method ${ }^{27}$ and a neural network interpolation to obtain the PESs in closed form for the six dimensions representing the molecular degrees of freedom. Ensuing extensive molecular dynamics (MD) simulations on the continuous PESs are then used to obtain the sticking probability as the proper statistical average over many trajectories with different initial molecular orientations and positions over the surface unit cell.

With this methodology, we first firmly establish that an adiabatic description based on the ground state BornOppenheimer $\mathrm{PES}^{28}$ can indeed not explain the experimentally reported low sticking probability of thermal molecules. Emphasizing the importance of spin-selection rules in the molecule-surface interaction, we model nonadiabatic effects by confining the trajectories of the impinging $\mathrm{O}_{2}$ molecules to motion on a PES, in which the spin-triplet character of the gas-phase molecule is conserved. This leads indeed to a significant reduction of the sticking probability at lower kinetic energies, in qualitative agreement with experiment. In order to further substantiate these findings of our previous work, ${ }^{25}$ we also critically discuss major uncertainties of our approach, which arise from confining the $\mathrm{O}_{2}$ trajectories exclu- 
sively on the spin-triplet constrained PES and from the employed approximate exchange-correlation (xc) functionals.

While this analysis reveals noticeable quantitative effects, it also clearly shows that spin-selection rules are indeed the ruling factor behind the reduced sticking probability for thermal molecules. Due to the hindered spin transition, the largest fraction of the impinging molecules is already repelled into the gas phase at rather large distances from the surface where other mechanisms such as charge transfer from the metal are still quite weak. Only for the remaining molecules such alternative mechanisms (and corresponding other electronic states) will start to play a role and will then most likely lead to dissociation.

\section{ROLE OF SPIN-SELECTION RULES FOR THE OXYGEN DISSOCIATION AT Al(111)}

When investigating the role of nonadiabatic effects, it is crucial to first define, what the adiabatic ground state of the system is and to what kind of nonadiabaticity one refers to. Central for the determination of the sticking probability are trajectories of impinging molecules, which start at a very large distance from the surface and, in the case of a successful dissociation event, end with two individual oxygen atoms. For large molecule-surface separations, the ground state of the system is given by an $\mathrm{O}_{2}$ molecule in its ${ }^{3} \Sigma_{g}^{-}$triplet ground state and a nonmagnetic $\mathrm{Al}(111)$ surface, whereas adsorbed at the surface the oxygen adatoms are as nonmagnetic as the $\mathrm{Al}(111)$ surface. Sometime during the molecular trajectory, the initial spin moment on the oxygen is therefore quenched, and in an adiabatic calculation, this happens gradually during the molecule's approach to the surface. In fact, we will see below that in adiabatic DFT calculations, this quenching occurs already partly when the molecule is still (infinitely) far apart, where wave functions do not yet overlap.

Interestingly, for an isolated $\mathrm{O}_{2}$ molecule, such a spin transition during dissociation would be forbidden by Wigner's spin-selection rules ${ }^{26}$ which state that in any elementary step of bond making or breaking, the total spin of the reactants and products must be the same. In other words, the spin of the reactants must be conserved or transferred to some other entity. In the case of the $\mathrm{O}_{2}$ dissociation at the $\mathrm{Al}(111)$ surface, this other entity to which the spin is transferred is the solid surface. However, if this transfer probability is still low at larger distances from the surface, the spin-selection rules imply that the probability for an electronic transition toward the $\mathrm{O}_{2}$ spin-singlet state $\left({ }^{1} \Delta_{g}\right)$ will be small, even if the surface potential shifts the ${ }^{1} \Delta_{g}$ energy below the spintriplet ${ }^{3} \Sigma_{g}^{-}$energy. Small probability means in this context a probability of similar magnitude as the spin-transfer probability in the gas phase, where the lifetime of spin-singlet $\mathrm{O}_{2}$ is $72 \mathrm{~min}$. Due to this low transition probability, the central assumption of the adiabatic picture may then become incorrect, which is that the electrons are at any time able to instantaneously follow the motion of the nuclei and remain in their electronic ground state.

Instead of molecular trajectories on the adiabatic PES, molecules conserving their initial spin-triplet character dur- ing the approach to the surface are then the relevant ones for the surface scattering and the determination of the sticking coefficient. This situation can be conveniently described in a diabaticlike picture, where the electronic structure at the $\mathrm{O}_{2}$ molecule is constrained to a certain spin state. ${ }^{27}$ Relevant nonadiabatic states of the interacting system correspond then to the ground and certain excited states of the system, when the $\mathrm{O}_{2}$ molecule is still so far away from the surface that there is no interaction. This would be a state corresponding to the $\mathrm{O}_{2}$ molecule in its ${ }^{3} \Sigma_{g}^{-}$spin-triplet gas-phase ground state and the $\mathrm{Al}(111)$ surface in its singlet ground state (henceforth, simply termed spin-triplet state). Another state corresponds to the $\mathrm{O}_{2}$ molecule in its ${ }^{1} \Delta_{g}$ spin-singlet state and the $\mathrm{Al}(111)$ surface in its singlet ground state (henceforth, termed spin-singlet state). Further constrained states include situations where electrons are transferred from the metal surface to the $\mathrm{O}_{2}$ molecule, e.g., the case of an $\mathrm{O}_{2}^{-}$ion and an $\mathrm{Al}(111)^{+}$surface (henceforth, termed ionic state).

In this nonadiabatic picture, the system dynamics is given by motion on and transitions between the various PESs corresponding to the different constrained states. At the beginning of the molecular trajectories, i.e., the largest moleculesurface distances, the energetic order of these PESs follows the excitation spectrum of the free $\mathrm{O}_{2}$ molecule: The spintriplet state is lowest in energy, followed by the spin-singlet state, which in experiment is about $1 \mathrm{eV}$ higher in energy. The next state of interest is the ionic state which is higher than the spin-triplet state by the difference of the $\mathrm{Al}(111)$ work function and the $\mathrm{O}_{2}$ electron affinity. Experimentally, this energy difference is about $4 \mathrm{eV}$ for an infinite separation between the molecule and the surface and is somewhat lowered by the Coulomb interaction at finite distances. The molecular trajectory starts in the spin-triplet state. At decreasing molecule-surface distances, the various constrained states are influenced differently by the surface potential, and eventually, the spin-singlet state will result as the lowest energy state.

If we focus for the moment on only the two energetically lowest constrained states at large distances, the situation is thus as shown schematically in Fig. 1. Since the adiabatic picture is not spin consistent, the adiabatic PES is lowered by a coupling of the spin-triplet and spin-singlet states, which is largest at the crossing point of the two spin-constrained PESs. Due to this lowering, the adiabatic PES can therefore be barrierless, even if the spin-triplet PES exhibits a barrier to dissociation, as shown in Fig. 1. In the adiabatic picture, the molecule would then dissociate along with a gradual quenching of its spin moment. However, if the crossing of the spin-triplet and spin-singlet PES happens at distances from the surface that are large enough to prevent an efficient spin transfer to the $\mathrm{Al}(111)$ surface, the spin-selection rules will prevent the transition from the spin-triplet to the spinsinglet state and the molecular motion will continue on the spin-triplet PES up to distances to the surface, where the increased coupling to the surface allows an efficient spin transfer. If the triplet PES then exhibits barriers, as shown schematically in Fig. 1, low energy molecules would not be able to overcome these barriers and would be reflected back into the gas phase, explaining the experimentally measured low sticking coefficient for thermal $\mathrm{O}_{2}$ molecules. 


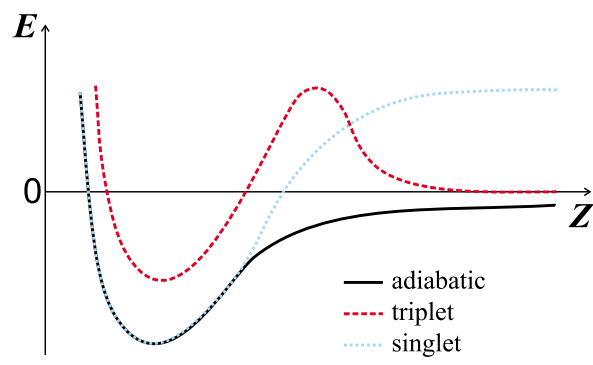

FIG. 1. (Color online) Schematic energy diagram showing the two spin constrained potential-energy surfaces (PESs) and the adiabatic PES as a function of the molecule-surface separation $Z$. The adiabatic ground state PES (solid line) has the lowest energy for all $Z$. Molecules travel initially on the spin-triplet PES (dashed line) and if this PES exhibits an energy barrier as shown here, lower energy molecules will be reflected, leading to a corresponding reduction in the sticking probability. The spin-singlet PES (dotted line) is an excited state at large $Z$, but becomes lower in energy for small $Z$.

Central for a validation of this conceptual understanding is therefore the computation of the spin-triplet PES. Important is not only whether this PES indeed exhibits barriers to dissociation but also at which molecule-surface distances these barriers are. The closer the barriers are located to the surface, the higher is the coupling to the surface and the lower the probability that the molecular motion will remain on the spin-triplet PES. In this respect, it is important to note that at small molecule-surface distances, also a multitude of other constrained states including the above discussed ionic state will become energetically favorable. While Fig. 1 focuses on only the two spin states for simplicity, it is then in principle the coupling and electronic transitions to all these states that will play a role. However, if the spin-triplet PES exhibits barriers at molecule-surface distances, where the energetic order of the PESs remains still largely as dictated by the gas-phase excitation spectrum, then only the spin-triplet state and at most transitions to the spin-singlet state are relevant for the determination of the sticking coefficient. A crucial first step is therefore to calculate, based on first principles, the spin-triplet PES and identify the size and location of possible energy barriers, as well as to analyze their implications for the sticking curve.

\section{METHODOLOGY}

\section{A. Calculation of the sticking curves}

The experimentally measured sticking coefficient is an average over a large number of molecule-surface collisions with different initial molecular configurations. These configurations comprise different molecular orientations with respect to the surface, as well as different lateral positions of the molecule's center of mass over the surface unit cell. A statistically reliable sticking curve can therefore only be calculated from a larger number of MD trajectories with random initial configurations that account for these degrees of freedom. We address this challenge by employing a divide and conquer approach. In this approach, the calculation of the sticking curve is split into three consecutive steps. ${ }^{29-33}$ In a first step, the PES of the molecule-surface interaction is mapped as a function of the relevant coordinates. In the second step, this precalculated mesh is then interpolated or fitted by a continuous representation. Such an interpolation of high-dimensional PESs is a tedious task, but in recent years, several techniques, such as analytical fits, ${ }^{31,34-37}$ tight binding representations, ${ }^{38-41}$ genetic programing, ${ }^{42}$ and the modified Shephard method, ${ }^{43,44}$ possibly combined with a corrugation-reduction scheme, ${ }^{33,45}$ have been developed. In the present work, we employ a very general neural network fitting scheme, which has already proven to be a powerful tool for the accurate representation of multidimensional PESs in similar applications. ${ }^{46-49}$ Since the evaluation of the energy and forces from the neural network representation is about 5 to 6 orders of magnitude faster than direct ab initio calculations, a large number of MD trajectories can be calculated in the last step of the "divide and conquer" approach to obtain the sticking probabilities at various molecular kinetic energies.

\section{B. Calculation of the potential-energy surfaces}

All DFT calculations have been carried out with the fullpotential all-electron code $\mathrm{DMOL}^{3}$ using numerical atomic orbitals as basis functions. ${ }^{50,51}$ For the oxygen atoms, the socalled all basis is used (19 atomic orbitals per atom), and for the aluminium atoms, the $d n d$ basis is applied (18 atomic orbitals per atom). These basis sets are essentially equivalent to a "double numeric" basis, i.e., the valence orbitals are described by a linear combination of atomic orbitals of the free atom and of the free positive ion, which is further improved by a set, of polarization functions. For details of the basis set we refer to Ref. 50. A real-space cutoff of $9 \mathrm{bohr}$ has been applied to the basis functions. The irreducible wedge of the first Brillouin zone is sampled by ten $k$ points. To improve convergence, a Pulay mixing scheme ${ }^{52,53}$ has been employed combined with a thermal Fermi broadening of $0.1 \mathrm{eV}$, extrapolating the energies to zero temperature. ${ }^{54}$ To check on the dependence of the obtained results on the exchange-correlation functional, the full PESs have been calculated using two different functionals, the $\mathrm{PBE}^{55}$ and the $\mathrm{RPBE}^{56}$ functionals. Even though both functionals are based on the generalized gradient approximation (GGA) and provide a much better description of the oxygen molecule than the local-density approximation ${ }^{57}$ (LDA), they yield rather different binding energies of the free oxygen molecule, as shown in Table I. In this respect, we use both functionals to obtain a first idea about the uncertainties in our results with respect to the description of electronic exchange and correlation. Compared to experiment, all functionals included in Table I yield a strong overestimation of the binding energy of the oxygen molecule. The reason for this overbinding has been described in detail by Gunnarsson and Jones ${ }^{58}$ and is due to an insufficient error compensation between the oxygen atom and the molecule, as the different nodal structures of the wave functions of these two species are not fully taken into account in the description of the exchange energy in jellium based exchange-correlation functionals (e.g., LDAs 
TABLE I. Binding energies $E_{b}$ and bond lengths $r$ of the free $\mathrm{O}_{2}$ molecule in its ${ }^{3} \Sigma_{g}^{-}$ground state, as obtained with our present computational setup and different exchange-correlation functionals. The zero point energy of about $0.1 \mathrm{eV}$ has not been taken into account, but is part of the experimental results.

\begin{tabular}{lcc}
\hline \hline Functional & $\begin{array}{c}E_{b} \\
(\mathrm{eV})\end{array}$ & $\begin{array}{c}r \\
(\AA)\end{array}$ \\
\hline $\mathrm{LDA}^{\mathrm{a}}$ & 7.43 & 1.21 \\
$\mathrm{PBE}^{\mathrm{b}}$ & 6.10 & 1.22 \\
PW91 $^{\mathrm{c}}$ & 6.07 & 1.22 \\
BLYP $^{\mathrm{d}}$ & 5.75 & 1.24 \\
RPBE & 5.65 & 1.23 \\
Expt. & $5.12^{\mathrm{f}}$ & $1.21^{\mathrm{g}}$ \\
\hline \hline
\end{tabular}

${ }^{a}$ Reference 57.

${ }^{\mathrm{b}}$ Reference 55 .

${ }^{\mathrm{c}}$ Reference 59.

${ }^{\mathrm{d}}$ Reference 60 .

${ }^{\mathrm{e}}$ Reference 56.

${ }^{\mathrm{f}}$ Reference 61.

gReference 62.

and GGAs). The consequences of this error in the molecular binding energy on our study of the dissociation process will be discussed in more detail in Sec. IV A.

The $\mathrm{Al}(111)$ surface is modeled by a $(3 \times 3)$ slab geometry consisting of seven aluminium layers. Tests with different supercells have shown that for the often employed (2 $\times 2$ ) supercells, the interaction energy between the oxygen molecules in neighboring cells is still about $0.3 \mathrm{eV}$ for an extended, but in the present context still relevant molecular bond length of $2.4 \AA$ in a parallel orientation to the surface. In the $(3 \times 3)$ cells, for comparison, this interaction is reduced to about $70 \mathrm{meV}$ for this particular bond length. Since the clean $\mathrm{Al}(111)$ surface shows only a marginal outward relaxation of about $1 \%$ of the interlayer distance, ${ }^{63-65}$ all atoms have been fixed in their bulk positions. The aluminium lattice constants obtained with PBE and RPBE are very simi$\operatorname{lar}(4.05$ and $4.08 \AA)$, and the PBE lattice constant has therefore been used in all calculations. To avoid interactions between the periodically repeated slabs, a large vacuum of $30 \AA$ has been used. This does not increase the computational effort due to the finite range of the localized basis functions but enables us to perform calculations for molecule-surface separations of up to $10 \AA$. Making use of inversion symmetry oxygen is adsorbed at both sides of the slab to prevent dipole interactions between the slabs through the vacuum region. At the largest molecule-surface separations considered in this work, the $\mathrm{O}_{2}$ molecule is then still more than $10 \AA$ away from its periodic image impinging on the other side of the slab.

When keeping the nuclei in the $\mathrm{Al}(111)$ surface at fixed positions, the PES for the oxygen dissociation is still six dimensional due to the molecular degrees of freedom. The commonly employed coordinate system is a superposition of a Cartesian coordinate system for the center of mass of the oxygen molecule $(X, Y$, and $Z$, the latter being the direction perpendicular to the surface) and a spherical coordinate sys-

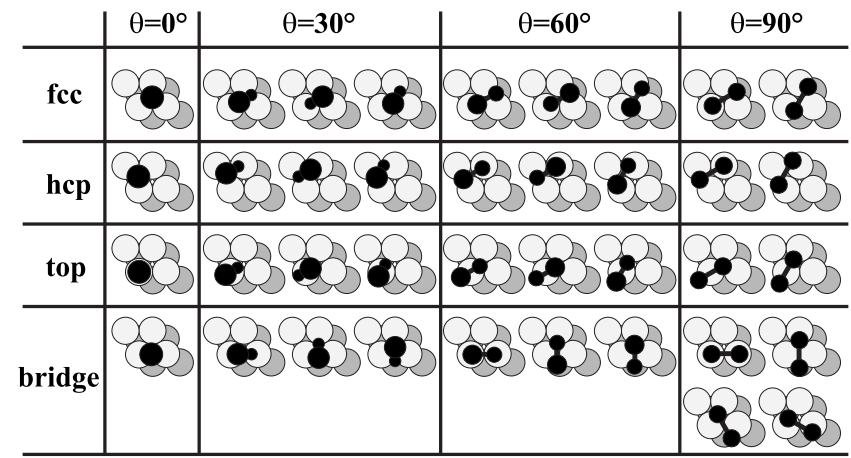

FIG. 2. Top views of the molecular configurations for which elbow plots have been calculated in the mapping of the sixdimensional potential-energy surface. For clarity, only four of the nine surface $\mathrm{Al}$ atoms in the $(3 \times 3)$ supercell are shown (gray spheres). The two oxygen atoms are shown as small black circles and the relative size of both atoms indicates their vertical height (larger sphere means further away from the surface and closer to the viewer). Correspondingly, only one atom is visible in configurations, in which the molecule approaches in a vertical orientation. $\theta$ is the angle between the surface normal and the molecular axis.

tem for the oxygen-oxygen bond length $r$, the angle between the molecular axis and the surface normal $\theta$, and the angle $\phi$ between the projection of the molecular axis in the $x y$ plane and the positive $x$ axis. The six-dimensional PES is mapped by calculating a number of two-dimensional cuts, in which the energy is given as a function of $r$ and $Z$ and which are commonly called "elbow plots" due to their characteristic shape. All other degrees of freedom, i.e., $\theta, \phi, X$, and $Y$, are fixed in a single elbow plot, and the configuration space referring to these four dimensions is mapped by calculating elbow plots for many different surface sites and many different molecular orientations. In the case of the adiabatic PES, a total of 38 different elbow plots has been calculated for each xc functional and the molecular configurations are detailed in Fig. 2. The symmetry of the surface has been fully exploited, in that only energies for configurations within the irreducible wedge of the unit cell spanned by the fcc, hcp, and top site have been calculated, as explained in Fig. 3. The energy zero point has been defined as the total energy for an infinite separation between the surface and the molecule, with the latter at its equilibrium bond length. This bond length of the free $\mathrm{O}_{2}$ molecule as obtained with the different functionals is also compiled in Table I.

As will be apparent below, the spin-triplet PES exhibits barriers to dissociation, which depend strongly on the molecular orientation with respect to the surface. Since also the height of the barriers depends strongly on the molecular orientation toward the surface, a more detailed mapping of the barrier region than in the adiabatic case was done to ensure an accurate description of the PES for the MD simulations. In addition to the full elbow plots at the high-symmetry fcc, hcp, top, and bridge sites used for the adiabatic PES, cf., Fig. 2, configurations with $r=1.3 \AA$ and $Z=2.1 \AA$ close to the maximum of the barrier have been calculated for all possible angular orientations of the molecule at the 11 "offsymmetry" sites shown in Fig. 3 by sampling the angles $\theta$ and $\phi$ in $30^{\circ}$ intervals. Consequently, the six-dimensional 


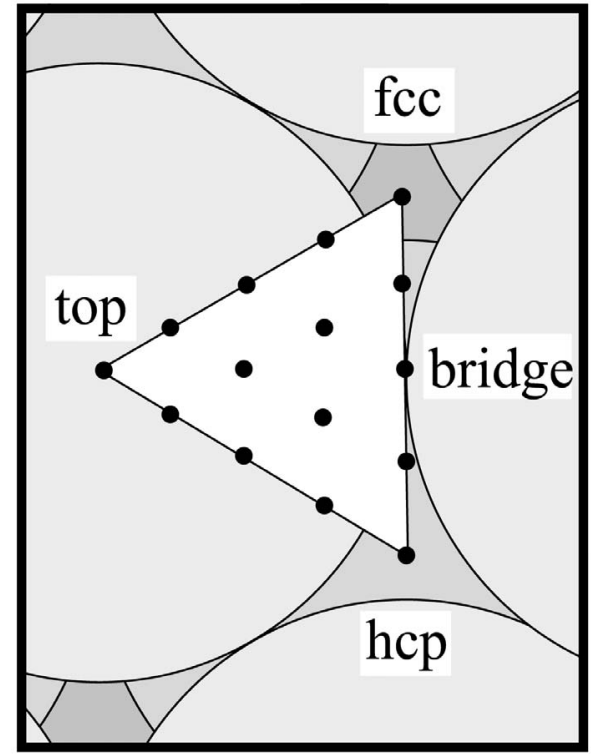

FIG. 3. Top view explaining the symmetry of the Al(111) surface. The irreducible wedge spanned by the fcc, hcp, and top sites is shown as white triangle. The surface atoms are represented by the grey circles. The surface sites within the irreducible part of the unit cell used for the mapping of the barrier region of the spin-triplet potential-energy surface are shown as black dots.

shape of the energy barrier is mapped very accurately, which allows us to properly account for steering effects, if present, in the molecular dynamics simulations.

\section{Locally constrained density functional theory}

While the adiabatic ground state PES is accessible by standard (spin-polarized) DFT calculations, the calculation of spin-constrained PESs requires the introduction of appropriate constraints on the electronic configurations. In order to localize the triplet spin at the oxygen molecule for all molecule-surface separations, we developed and employed a locally constrained DFT technique in the spirit of the seminal work of Dederichs et al. ${ }^{67}$ which is also very similar to the independently developed approach by $\mathrm{Wu}$ and Van Voorhis. ${ }^{68}$ For a detailed description of this technique and a comparison to other approaches, we refer to Ref. 27 and present here only a concise summary.

The central idea in the LC-DFT technique employed in this work is to split the system into two subsystems, the oxygen molecule and the $\mathrm{Al}(111)$ surface, and to constrain the electron numbers of these subsystems according to the constrained state of interest. In the general case the calculations are spin polarized, and for the two subsystems, four electron numbers $N_{\mathrm{O}_{2}}^{\uparrow}, N_{\mathrm{O}_{2}}^{\downarrow}, N_{\mathrm{Al}}^{\uparrow}$, and $N_{\mathrm{Al}}^{\downarrow}$ have to be considered. These electron numbers are determined by projecting the Kohn-Sham states into the Hilbert spaces of the two subsystems, which in the case of a localized atomic orbital basis set are spanned by the basis functions centered at the atoms of the respective subsystems. This projection yields the partial densities of states of the four subsystems, which are then filled separately with the four electron numbers cor- responding to the nonadiabatic state of interest. In the general case, this yields four different Fermi energies $\epsilon_{F, \mathrm{O}_{2}}^{\uparrow}$, $\epsilon_{F, \mathrm{O}_{2}}^{\downarrow}, \epsilon_{F, \mathrm{Al}}^{\uparrow}$, and $\epsilon_{F, \mathrm{Al}}^{\downarrow}$, which are subsequently aligned under the constraint of fixed electron numbers. This alignment is achieved by the introduction of a configuration-dependent auxiliary potential $H_{A}^{\sigma}$. $H_{A}^{\sigma}$ is a projection operator into the oxygen subspace and thus allows to shift $\epsilon_{F, \mathrm{O}_{2}}^{\sigma}$ with respect to $\epsilon_{F, \mathrm{Al}}^{\sigma}$. The auxiliary potential is added to the standard DFT Hamiltonian $H_{0}^{\sigma}$ to yield a new effective Hamiltonian $H_{\mathrm{eff}}^{\sigma}$ for each spin $\sigma$,

$$
H_{\mathrm{eff}}^{\sigma}=H_{0}^{\sigma}+H_{A}^{\sigma} \text {. }
$$

The strength of $H_{A}^{\sigma}$ is adjusted self-consistently until the Fermi energies are aligned, i.e., the electronic structure problem is solved fully self-consistently under the constraint of fixed electron numbers in the two subsystems and for each spin channel.

The electronic structure of the system is thus completely relaxed under the given constraint (formulated as an additional external potential) and in this sense, the LC-DFT method calculates a "constrained electronic ground state" based on the Hohenberg-Kohn theorem. ${ }^{69}$ The method is very general, and by adapting the electron numbers in principle, arbitrary constrained PESs can be calculated for different spin and charge states of the subsystems. We stress, however, that this constraint does, of course, not allow us to overcome limitations related to the employed exchangecorrelation functional (which, e.g., affects the multiplet structure $^{70}$ of some systems) and we will come back to this point below.

\section{Neural network interpolation}

In this paper, we closely follow the neural network (NN) fitting procedure described in detail in Refs. 48 and 49 and summarize here only the features that will be relevant for the later sections. We only note that a different way of incorporating the symmetry of the surface into the NN has been used which no longer contains any approximations and which is described in detail elsewhere. ${ }^{71}$ Basically, this procedure consists of a coordinate transformation of the original six molecular coordinates to a set of symmetry functions, 11 in this case, that is used as input for the NN.

In general, a neural network is a nonlinear technique that allows us to fit any function to a high accuracy and does not require any knowledge about the functional form of the underlying problem. ${ }^{72}$ In particular, multilayer feed-forward neural networks have already successfully been employed to provide accurate fits of potential-energy surfaces. ${ }^{46-49}$ In the present work, this NN type is applied to fit a smooth and continuous function to the DFT data by optimizing a set of parameters. This function has an analytic form and consequently the forces required in the MD simulations can easily be obtained from the derivatives of this function. This way the energies and forces are consistent, which is essential for MD applications.

The general form of a small feed-forward $\mathrm{NN}$ is shown schematically in Fig. 4. It consists of several layers, each of which contains one or more nodes represented by the gray 


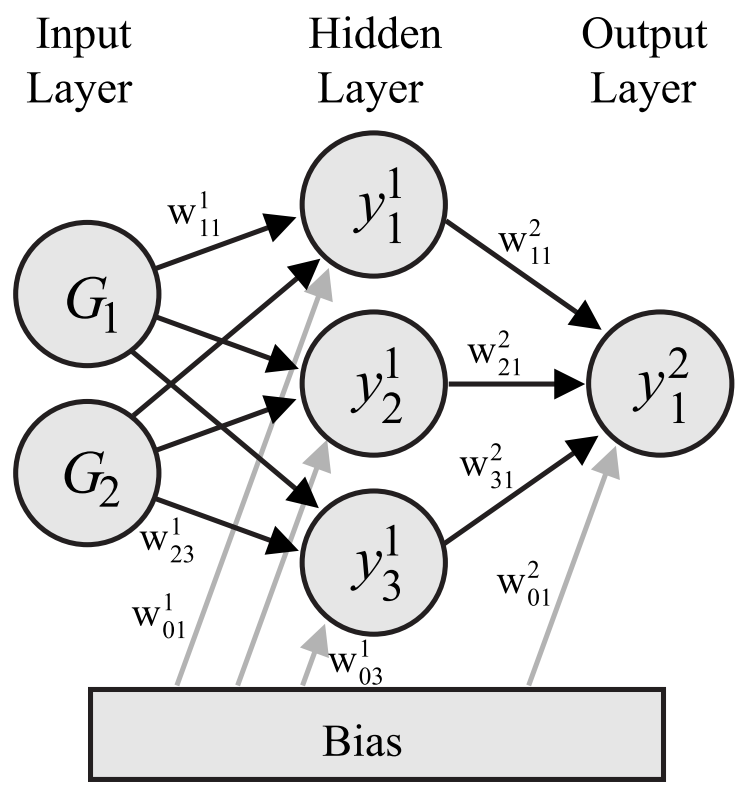

FIG. 4. Structure of a $\{2-3-1\}$ feed-forward neural network $(\mathrm{NN})$. The gray circles represent the nodes of the network. Each node $j$ in layer $k$ is connected to the nodes $i$ in the preceding layer by the weights $w_{i j}^{k}$, the parameters of the NN. The bias adds a constant to each node in the hidden and output layers to adapt the positions of the nonlinearity intervals of the activation functions. The output $y_{1}^{2}$ is the potential energy $E$ and is calculated according to Eq. (2).

circles. In the given example, two nodes in the input layer represent two arbitrary coordinates $G_{1}$ and $G_{2}$ determining the total energy $E$. This energy corresponds to the single node in the output layer. Between the input and the output layer, one hidden layer with three nodes is located. The term hidden layer is used because the numerical values at the nodes of this layer are just auxiliary quantities without a physical meaning. Each node $i$ of a certain layer is connected with the node $j$ in the subsequent layer via a weight parameter $w_{i j}^{k}$, where $k$ represents the index of the target layer. On each node, all the values from the nodes of the preceding layer are summed after being multiplied by the connecting weight. On the resulting sum, a nonlinear function $f_{a}$ is applied. This function is called activation function and is typically a sigmoidally shaped function that introduces the nonlinearity capability to the NN. Specifically, we use the hyperbolic tangent as activation function in this work. For very large or very small arguments, the activation functions converge to a constant number, but for a certain interval, the output changes in a nonlinear way allowing to fit very complex functions. The bias unit acts as an adjustable offset to adapt the position of the nonlinearity interval of the activation functions. Then, the obtained function value of each node is passed to all the nodes of the subsequent layer and multiplied by the corresponding connecting weights. In the output layer, the values are collected and the output value is calculated by applying a linear function as activation function. In a feed-forward NN information is transferred only in one direction through the network, from the input layer via the hidden layer(s) to the output layer. In general, each layer including input and output layers can contain many more nodes than shown in the simple example in Fig. 4, and also more than one hidden layer is typically used.

With this construction, a complicated nonlinear function relating the input coordinates to the energy is defined. This function can be given analytically and it depends on many parameters, the weights $w_{i j}^{k}$. For the example, given in Fig. 4, this function is

$$
E(\mathbf{G})=f_{a}^{2}\left[w_{01}^{2}+\sum_{j=1}^{3} w_{j 1}^{2} f_{a}^{1}\left(w_{0 j}^{1}+\sum_{i=1}^{2} w_{i j}^{1} G_{i}\right)\right],
$$

where $y_{i}^{k}$ labels the value of node $i$ in layer $k$, and for the input nodes, we have used the symbol $\mathbf{G}=\left\{G_{i}\right\} . w_{0 j}^{k}$ is the bias weight for the activation function $f_{a}^{k}$ acting on node $j$. In this work, we use a short hand notation to describe the structure of a neural network. The example in Fig. 4 is a $\{2-3-1-t l\}$ network, i.e., two nodes in the input layer, three nodes in one hidden layer, and one node in the output layer. " $t$ " indicates the use of a hyperbolic tangent as activation function in the hidden layer, while a linear "l" function has been used in the output layer.

In order to calculate the output, i.e., the interpolated energy, the connecting weight parameters have to be known. They are determined by training the NN using a set of known DFT data points. An independent test set of DFT data, which has not been used in the parameter optimization is used to check the accuracy of the fit for geometries not included in the training set. For the adiabatic PES computed with the PBE functional, 1723 DFT energies were calculated and split into a training and a test set. The test set contains 79 randomly chosen points and is not used for the optimization of the network parameters. After extensive testing, the best fit was achieved with a $\{11-38-38-1-t t l\}$ NN structure. The mean average deviations (MADs) with respect to the original DFT data are 38 and $62 \mathrm{meV}$ in the training and test set, respectively. The root mean squared error (RMSE) of the full training set is $0.080 \mathrm{eV}$; for the test set, it is $0.105 \mathrm{eV}$. To obtain a good fit particularly in the region of the PES that is accessible in the MD runs, i.e., the regions with a potential energy of less than $+1 \mathrm{eV}$ with respect to the sum of the isolated subsystems, in all fits reported in this work gradually higher fitting weights have been assigned to points close to the minimum energy path. This reduces the RMSE along the minimum energy path in the entrance channels of the elbow plots to a few $\mathrm{meV}$ and reduces also significantly the maximum deviation we obtain for the data points that are fitted worst. While this maximum fitting error is with $0.69 \mathrm{eV}$ still sizeable for the data set with energies up to $+1 \mathrm{eV}$, only 18 out of the 704 data points that correspond to the entrance channel of an impinging thermal $\mathrm{O}_{2}$ molecule in a MD simulation exhibit errors that are larger than $50 \mathrm{meV}$. We carefully checked these few single data points with high fitting error to make sure that they do not introduce artificial topological features to the PES or affect the sticking curve simulations (which are most sensitive to the PES barriers). A similarly good fit has been achieved for the adiabatic PES computed with the RPBE functional. Here, we calculated 1369 DFT energies, and the test set consisted of 69 randomly 
chosen points. The training and test MADs obtained with a \{11-40-40-1-ttl $\}$ network are 22 and $50 \mathrm{meV}$, and the RMSE are 0.041 and $0.100 \mathrm{eV}$, respectively. For both xc functionals, all calculated elbow plots are perfectly reproduced by the NN fits.

Due to the more complicated, barrier-containing topology of the spin-triplet PES, we used a larger number of DFT data points for the NN interpolation. Specifically, 2870 and 2917 DFT energy points were computed for the RPBE and the PBE xc functionals, respectively. For the interpolation, 96 of the RPBE energies and 97 of the PBE energies were chosen randomly as test data set. The best fit for the RPBE data points is obtained from a $\{11-40-40-1-\mathrm{ttl}\} \mathrm{NN}$ and have MADs of 0.023 and $0.033 \mathrm{eV}$ for the training and test points, respectively. The corresponding RMSEs are 0.049 and $0.070 \mathrm{eV}$. In the case of the PBE PES, the MADs are 0.035 and $0.031 \mathrm{eV}$ and the RMSEs are $0.078 \mathrm{eV}$ and $0.050 \mathrm{eV}$, respectively, obtained also with a $\{11-40-40-1-\mathrm{ttl}\} \mathrm{NN}$. Again, we found the biggest error in a single data point in potential-energy regions up to $+1 \mathrm{eV}$ to be still relatively large ( $0.43 \mathrm{eV}$ for RPBE), but due to the employed fitting weights, this largest error in the data points that are accessible by a thermal $\mathrm{O}_{2}$ molecule is reduced to $17 \mathrm{meV}$ in the case of the RPBE PES. With the ultimately achieved fits, all 38 elbow plots and also the additional points at the offsymmetry sites are accurately reproduced. Further details of the fitting procedure and an error analysis are published elsewhere. ${ }^{71}$

\section{E. Molecular dynamics simulations}

The calculation of a statistically reliable sticking curve requires the calculation of molecular trajectories with different initial configurations (initial lateral position and orientation of the molecule with respect to the surface). The MD simulations are based on solving Hamilton's equations of motion,

$$
\begin{gathered}
\dot{q}_{i}=\frac{\partial H}{\partial p_{i}}, \\
\dot{p}_{i}=-\frac{\partial H}{\partial q_{i}},
\end{gathered}
$$

with the classical nuclear Hamiltonian

$$
\begin{aligned}
H= & \frac{1}{2 M_{\mathrm{O}_{2}}}\left(p_{X}^{2}+p_{Y}^{2}+p_{Z}^{2}\right) \\
& +\frac{1}{2 \mu}\left(p_{r}^{2}+\frac{p_{\theta}^{2}}{r^{2}}+\frac{p_{\phi}^{2}}{r^{2} \sin ^{2} \theta}\right)+V(\mathbf{R}, \mathbf{r}),
\end{aligned}
$$

where $p$ is the momentum, $M_{\mathrm{O}_{2}}$ the mass, and $\mu$ the reduced mass of the oxygen molecule. $V(\mathbf{R}, \mathbf{r})$ is our NN representation of the potential energy. For the numerical integration, we employ a Burlisch Stoer algorithm ${ }^{73}$ with a variable time step to improve the efficiency and accuracy.

Initially, the oxygen molecule has the equilibrium gasphase bond length and is placed $9.5 \AA$ above the surface. The angular orientation of the molecule and the lateral position are chosen randomly. The initial velocity of the molecule toward the surface is determined by the translational kinetic energy and the lateral velocity components are zero resulting in a perpendicular angle of incidence of the molecule as in experiment. ${ }^{7}$ The trajectories are assumed to yield a dissociation event, if the $\mathrm{O}_{2}$ bond length is stretched beyond $2.4 \AA$, i.e., the bond length has doubled, and the momentum $p_{r}$ is still positive. We have verified that the specifics of this dissociation criterion plays no role for the sticking curves discussed in this work. Specifically, the lowering of the triplet sticking curve results from those molecules directly repelled at the triplet PES barriers described below. Molecules that overcome these barriers will predominantly dissociate, and a discussion of the specific mechanisms behind this is outside the scope of the present work. The molecule is considered as reflected, if $Z$ exceeds $6 \AA$ with a positive $p_{Z}$, i.e., the molecule is leaving the surface. The sticking curve is determined for kinetic energies ranging from 0.025 to $0.975 \mathrm{eV}$ in steps of $50 \mathrm{meV}$. From tests for the spin-triplet constrained case (see below), we found that the qualitative form of the sticking curve was already obtained, when averaging over around 20 trajectories with random initial $\mathrm{O}_{2}$ orientation for each kinetic energy. The error in the sticking probability $S$ for $N$ trajectories is given by $\sqrt{\frac{S(1-S)}{N}}$. Since the MD runs on the interpolated NN-PESs are computationally inexpensive, the fully converged sticking curves presented here were obtained by averaging over 2000 trajectories for each kinetic energy.

\section{RESULTS}

\section{A. Adiabatic picture}

\section{Adiabatic potential-energy surface}

We begin our study with the adiabatic PES for the oxygen dissociation at $\mathrm{Al}(111)$. Three of the calculated elbow plots are shown in Fig. 5 for both the RPBE and the PBE functionals. In the case of the RPBE functional, we find several elbow plots with shallow energy barriers of up to $0.1 \mathrm{eV}$, while in the case of the PBE functional, none of the calculated elbow plots shows a barrier toward dissociative adsorption. These results are in good agreement with previous calculations of parts of this PES in smaller surface unit cells. ${ }^{11-13}$ We only mention in passing that there is a general problem when calculating the adiabatic PES for the dissociation of oxygen at $\mathrm{Al}(111)$ with standard DFT implementations. There is a charge transfer from the metal to the oxygen molecule that is present even for an infinite molecule-surface separation. This is because the antibonding $2 \pi^{* \downarrow} \mathrm{O}_{2}$ orbital, which is unoccupied in the free molecule, is lower in energy than the Fermi level of the metal surface. Consequently, electron density is transferred from the surface to the molecule, which raises the energy of the $2 \pi^{* \downarrow}$ orbital until it is aligned with the Fermi level. This charge transfer to the molecule is of the order of 0.01e for large, even macroscopic distances from the surface, as has been discussed in detail in Ref. 27.

A direct comparison of the elbow plots obtained with the RPBE and the PBE functional shows that the RPBE PES is less attractive, which is a typical feature of this functional and is consistent with previous adsorption studies. ${ }^{56}$ How- 
(a)

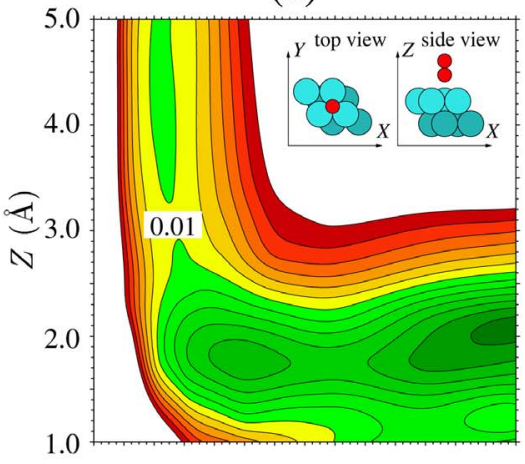

(d)

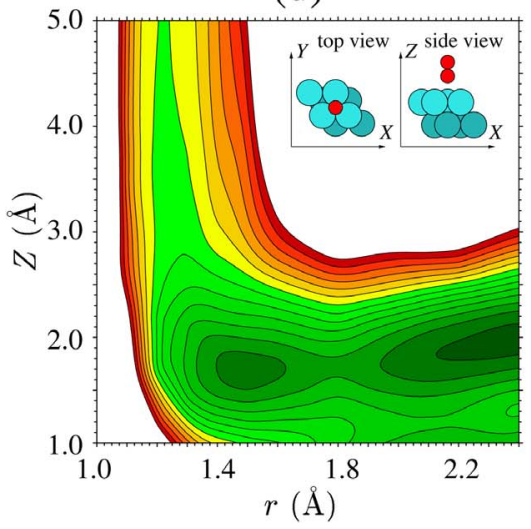

(b)

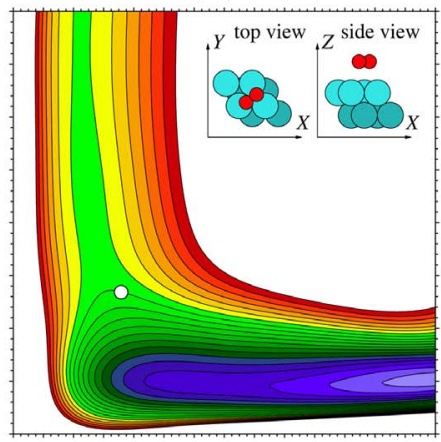

(e)

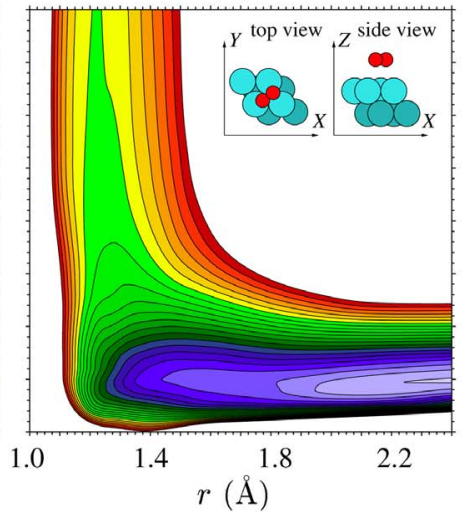

(c)

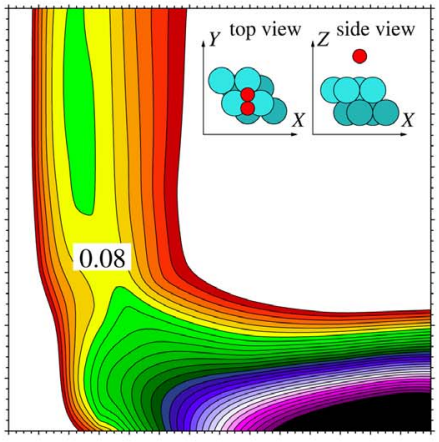

(f)

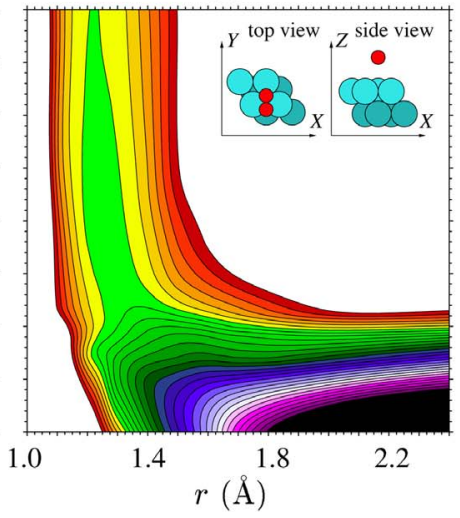

FIG. 5. (Color online) Two-dimensional cuts ("elbow plots") through the six-dimensional adiabatic PES for oxygen dissociation at the $\mathrm{Al}(111)$ surface. The energy is shown as a function of the center-of-mass distance of the molecule from the surface $Z$ and the oxygen-oxygen bond length $r$. In (a), (b), and (c), the elbow plots obtained with the RPBE functional are shown for the three different molecular orientations explained in the insets. In (d), (e), and (f), the corresponding elbow plots obtained from the PBE functional are shown. Energy barriers are labeled in eV. The energy difference between the contour lines is $0.2 \mathrm{eV}$.

ever, the energy difference between the PBE and the RPBE PES is far smaller than the $0.5 \mathrm{eV}$ binding energy difference of the free $\mathrm{O}_{2}$ molecule obtained with these functionals, cf. Table I. This indicates that the strong overbinding of the $\mathrm{O}_{2}$ molecule does not carry through to the shape of the adiabatic PES. The latter is instead determined by energy differences of different molecular configurations, which are likely to have similar errors in the xc energy.

When the oxygen molecule approaches the surface, the energetically more favorable orientation is initially perpendicular to the surface in agreement with previous studies. ${ }^{12,13}$ Closer to the surface the parallel configuration is preferred, which allows a stronger interaction of both atoms with the surface. During the approach to the surface, there is a continuous charge transfer from the surface to the molecule and a continuous reduction of the triplet spin until finally the singlet state of the adsorbed adatoms is adopted. The oxygen dissociation is a strongly exothermic process and the final binding energy of an oxygen atom with respect to a free $\mathrm{O}_{2}$ molecule is about $4.1 \mathrm{eV}(\mathrm{PBE})$ and $3.8 \mathrm{eV}$ (RPBE) in the employed $(3 \times 3)$ supercell. At higher coverages, this even increases due to attractive oxygen-oxygen interactions, ${ }^{65}$ i.e., from an energetic point of view, both abstraction and dissociative chemisorption of both atoms are possible.

\section{Adiabatic sticking curve}

Due to the almost complete absence of energy barriers in the computed adiabatic PES (for both xc functionals), a high sticking probability is very likely to be found. Nevertheless, slow molecules might be dynamically steered to certain surface sites and angular orientations which can have a strong effect on the sticking properties. ${ }^{31}$ It is not possible to assess such effects by a mere inspection of low-dimensional cuts of the PES, and we correspondingly carried out extensive MD simulations on the interpolated PES. For both xc functionals, the sticking probability was found to be basically unity independent of the kinetic energy, which is in strong disagreement with experiment, as shown in Fig. 6. In case of the PBE functional, this is immediately obvious due to the complete absence of energy barriers toward dissociation. Yet, also the shallow energy barriers found for some elbow plots calculated with the RPBE functional do not reduce the sticking probability. This has two reasons. First, the height of these barriers is very small so that they can be overcome even by thermal molecules in most cases. Second, slow molecules, which are most likely to be stopped by the barriers, are steered ${ }^{66}$ toward the barrier-free entrance channels.

Careful tests to determine the dependence of the sticking curves on the actual NN fit and the number of MD trajecto- 


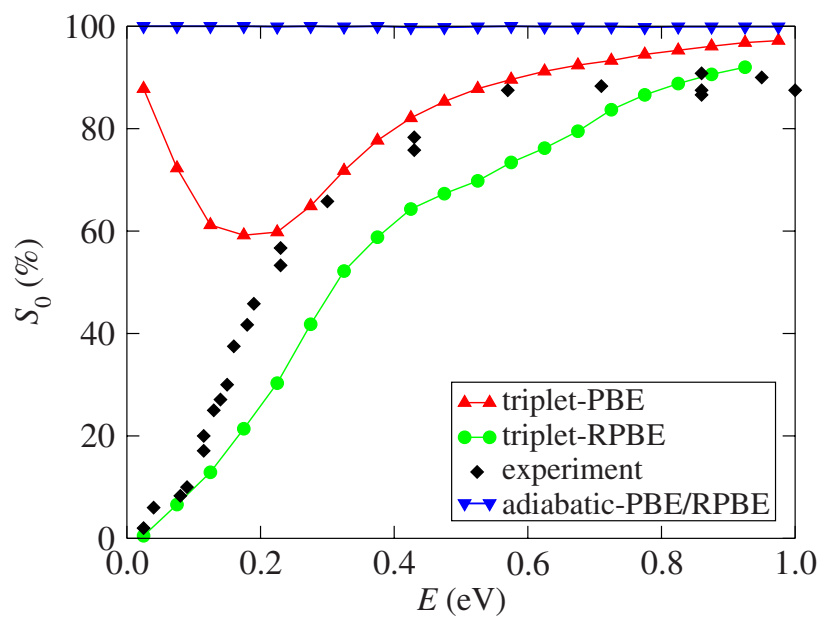

FIG. 6. (Color online) Sticking curves obtained from MD simulations on the adiabatic and the spin-triplet potential-energy surfaces as calculated with two different exchange-correlation functionals (PBE and RPBE). The experimental data (solid diamonds) are taken from Ref. 7. $E$ is the translational kinetic energy of the oxygen molecules.

ries have been carried out, all with the same result; the sticking probability is essentially $100 \%$ for thermal molecules. The identical result obtained with two xc functionals that yield a largely different $\mathrm{O}_{2}$ binding energetics, cf., Table I, renders it somewhat unlikely that the accuracy of the employed GGA functionals is insufficient for the description of the dissociative adsorption process. Finally, we also performed 24 on-the-fly ab initio $\mathrm{MD}$ runs for thermal $\mathrm{O}_{2}$ molecules, where the full dynamics of the $\mathrm{Al}$ atoms was taken into account. In all cases, the $\mathrm{O}_{2}$ molecule dissociated and the bond length was already notably weakened at moleculesurface distances, where significant O-induced movement of the $\mathrm{Al}$ atoms had not even set in. This suggests that neither the rigid substrate approximation underlying our approach may be held responsible for the dramatic disagreement with the experimental data. We therefore have to conclude that the low sticking probability of thermal oxygen molecules impinging on the $\mathrm{Al}(111)$ surface cannot be explained by calculations based on the adiabatic PES obtained from state-ofthe-art DFT.

\section{B. Nonadiabatic picture}

\section{Spin-triplet potential-energy surface}

If we therefore turn to the role of nonadiabatic effects, the discussion in Sec. II suggests to focus on the question of whether the spin-triplet constrained PES exhibits dissociation barriers and at which height over the surface they are located. A straightforward approach to this crucial PES would be to constrain the overall system to a spin-triplet configuration by means of the fixed spin moment ${ }^{74}(\mathrm{FSM})$ technique. Here, no constraint on the spatial distribution of the magnetization density is made, and only the overall numbers of spin-up and spin-down electrons in the system are fixed. We computed the PES with this approach, but obtained barrierless PESs that very much resemble the adiabatic ones. The reason is that in the FSM calculations close to the surface the triplet spin is becoming more and more delocalized. For $Z<1.8 \AA$, the triplet spin is in fact almost completely delocalized in the interior of the aluminium slab and almost no magnetization density is left on the oxygen molecule, as illustrated in Fig. 7. Since this is not the physics we want to describe, a different approach to the spin-triplet PES is required that locally confines the magnetization density to the oxygen molecule.

We achieve this confinement with the LC-DFT approach ${ }^{27}$ that allows us to control the electron numbers in the oxygen and the aluminium subsystems for each spin explicitly. Using this method, the spin-triplet PES has been calculated employing the PBE and the RPBE functionals. ${ }^{75}$ In Fig. 8, three of the 38 calculated elbow plots are shown for both functionals. In contrast to the adiabatic PES sizeable energy barriers exist with heights up to $0.9 \mathrm{eV}$. This finding is thus at variance with the calculations of Hellman et al. ${ }^{19}$ Although their so-called "neutral diabatic" PES calculated with the similar Perdew-Wang xc functional ${ }^{59}$ should be (at least in the region of the presently identified energy barriers) equivalent to our spin-triplet PBE PES, no clear energy barriers were found in their work. As already discussed in detail in Ref. 27, this is most likely due to shortcomings of the $\triangle \mathrm{SCF}$ type approach employed by Hellman et al.

In the RPBE case, there are barriers for all configurations, the lowest being about $0.05 \mathrm{eV}$ for a molecule oriented parallel to the surface above a bridge site with $\phi=0$ (not shown). The typical stronger binding of the PBE functional reduces the barriers in the PES computed with this functional by about $0.3-0.4 \mathrm{eV}$ compared to the RPBE case. For six of the 38 calculated elbows, the barrier disappears therefore even completely (all for parallel molecular orientations). Such a pronounced functional dependence has not been found in the adiabatic PES, and we will see below the consequences this has on the calculated sticking curve. Apparently, the exchange part, which is the only difference between the PBE and the RPBE functional ${ }^{56}$ is particularly sensitive to the triplet configuration close to the surface, a region where most or all of the spin has vanished in the adiabatic calculations.

In order to understand the origin of the energy barrier, it is instructive to analyze the magnetization density at the energy barrier. This is shown in Fig. 7 for a molecule parallel to the surface above an fcc site for the RPBE functional. In (a), the magnetization density of a free oxygen molecule in its spintriplet ground state without the metal surface is shown for comparison. In (b), the strongly reduced magnetization density of an adiabatic calculation is given. The integrated adiabatic magnetization density amounts to $0.064 \mu_{B}$ instead of $2.0 \mu_{B}$ for the ideal triplet. The magnetization density in (c) corresponds to the triplet FSM calculation. Although the integrated magnetization density still corresponds to a full triplet, a large amount of the spin has been transferred to the aluminium slab, which is the reason why the FSM method does not yield energy barriers. In (d), the magnetization density for the triplet LC-DFT calculation is plotted. The triplet spin is localized at the oxygen molecule causing a depletion of spin-up density in the surrounding region of the metal 


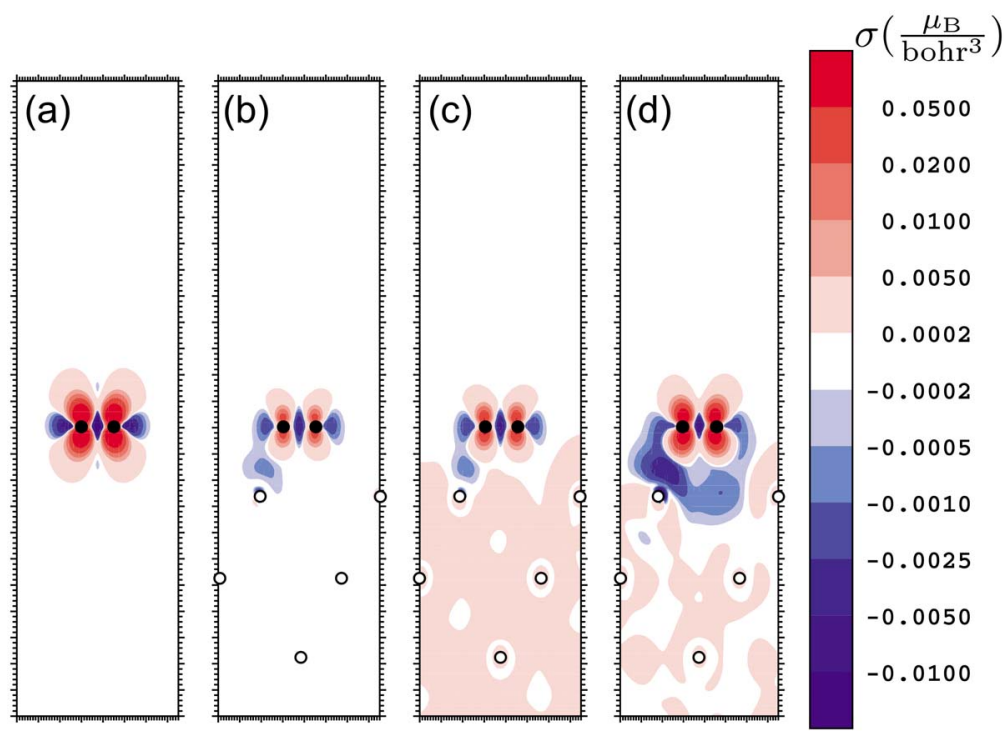

FIG. 7. (Color online) Magnetization-density plot obtained with the RPBE functional and for a molecule with a bond length of $1.3 \AA$, oriented parallel to the surface and $2.1 \AA$ above the fcc site. This molecular configuration corresponds to a position on the triplet energy barrier [indicated by the white circle in Figs. 5(b) and 8(b)]. Shown is a side view, with the upper half corresponding to the vacuum and the lower half to the $\mathrm{Al}$ surface (white circles represent the positions of $\mathrm{Al}$ atoms in the shown cut plane, and black circles the positions of the two $\mathrm{O}$ atoms). In (a), the magnetization density of a free $\mathrm{O}_{2}$ molecule in its triplet ground state without the aluminium slab is shown. (b) gives the reduced magnetization density in the adiabatic calculation. In (c), the magnetization-density distribution for a triplet fixed spin moment calculation is shown, while (d) refers to a triplet constrained calculation.

surface because of the Pauli repulsion of like spins. This increased Pauli repulsion is thus the origin of the energy barrier. As the metal surface is in an overall singlet state, the displaced aluminium spin-up density is delocalized in the slab, but in contrast to the FSM calculation, no net magnetization of the slab is present.

\section{Spin-triplet sticking curve}

MD simulations have been carried out on the spin-triplet PES for both xc functionals. These simulations are complementary to the MD trajectories on the adiabatic PES, in that the latter represent the case of an instantaneous charge transfer and spin reduction when the molecule approaches the surface, while for the MD confined to the triplet PES, no charge transfer and no change in the spin are allowed at any time. This corresponds therefore to the limit of an infinite lifetime of the molecule on the spin-triplet PES, which is a good approximation if the motion of the molecule is fast compared to the probability for electronic transitions. The resulting sticking curves are shown in Fig. 6. The sticking curve based on the PBE triplet PES is very similar to the RPBE curve for medium and high kinetic energies. The upshift of the PBE sticking coefficient with respect to the RPBE values can be explained by the systematically lower energy barriers in case of the PBE functional and provides a kind of "functional error bar." For low kinetic energies, however, the PBE sticking curve deviates qualitatively from the RPBE curve and the experimental data. The reason for the high sticking probability of about $87 \%$ for thermal molecules in this case is the absence of energy barriers for a few molecular configurations. Thermal molecules approach the sur- face very slowly and have sufficient time to adapt their orientation and lateral position to the shape of the surface potential. The majority of the lowest energy molecules is therefore steered into the few barrier-free entrance channels and can adsorb at the surface, whereas this dynamical steering becomes less efficient with increasing kinetic energy of the molecules.

The difference in the sticking probabilities of thermal molecules obtained with the two xc functionals shows how critical smallest energy differences in the PES can be for the resulting sticking curve. Although the PBE triplet PES is clearly less attractive than the adiabatic PES, in the typical barrier region the energy of the few barrierless configurations is only about $20 \mathrm{meV}$ lower than the vacuum level. Very small energy increases in this region would therefore be sufficient to yield energy barriers. In fact, if the energy were only by about $50 \mathrm{meV}$ higher, both functionals would yield qualitatively the same sticking curve. Although there is thus a pronounced $\mathrm{xc}$ functional dependence, a significant reduction of the sticking probability is nevertheless obtained for both functionals. This reduction is not present in the adiabatic case, no matter which functional is used.

Finally, we again employed on-the-fly ab initio MD simulations to check on the validity of the rigid substrate approximation underlying our approach. In Fig. 9, the vertical positions of the two oxygen atoms in the course of a trajectory are shown for a molecule oriented perpendicular to the surface above an fcc site. The dotted lines represent the positions of the oxygen atoms as obtained from the NN-based MD employing the frozen-surface approximation. The molecule has an initial kinetic energy of $0.15 \mathrm{eV}$, which is not sufficient to overcome the barrier, and consequently, it is 
(a)

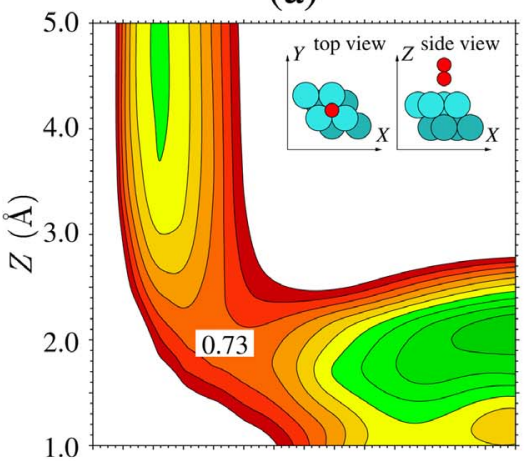

(d)

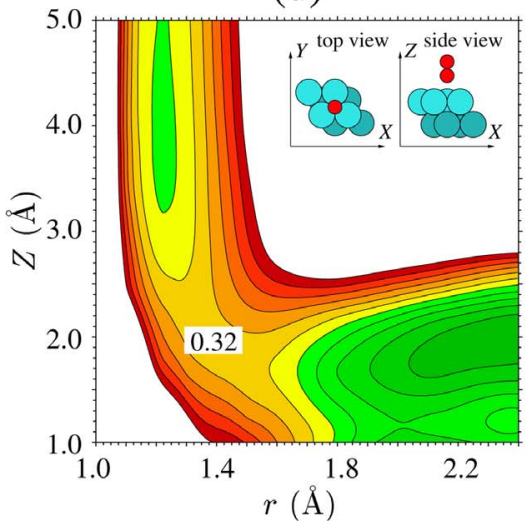

(b)

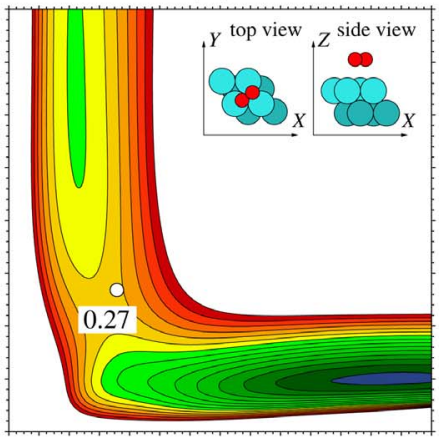

(e)

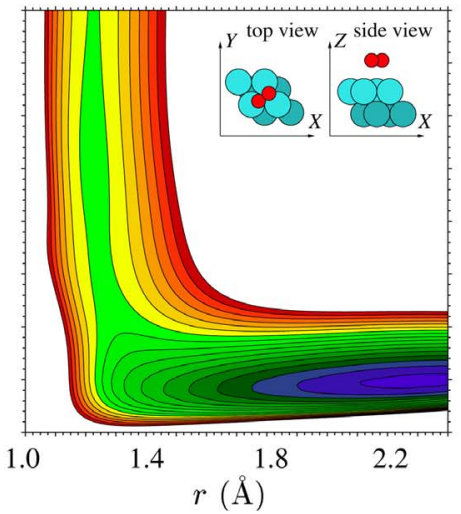

(c)

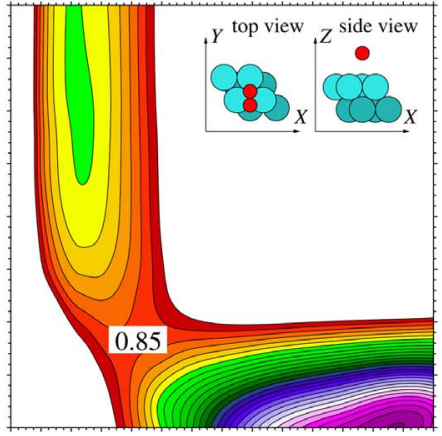

(f)

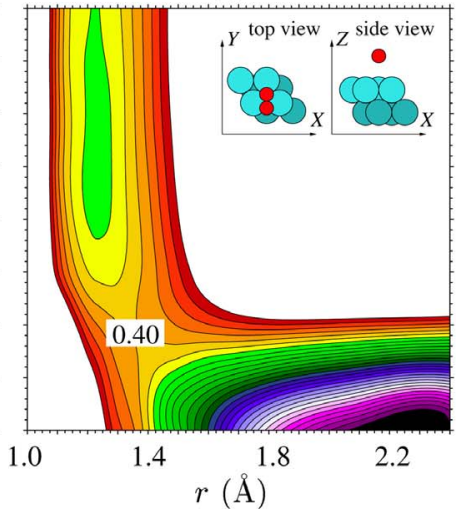

FIG. 8. (Color online) Two-dimensional cuts (elbow plots) through the six-dimensional spin-triplet PES for the oxygen dissociation at the $\mathrm{Al}(111)$ surface obtained with the locally-constrained DFT approach. Equivalent to Fig. 5, the energy is shown as a function of the center-of-mass distance of the molecule from the surface $Z$ and the oxygen-oxygen bond length $r$. In (a), (b), and (c), the elbow plots obtained with the RPBE functional are shown for the three different molecular orientations explained in the insets. In (d), (e), and (f), the corresponding elbow plots obtained from the PBE functional are shown. Energy barriers are noted in eV. The energy difference between the contour lines is $0.2 \mathrm{eV}$.

reflected back into the gas phase. The straight black lines represent the oxygen positions in a corresponding direct $a b$ initio MD run for the same initial atomic positions. The trajectory of the molecule is hardly changed. The induced motion of the aluminium atoms is very small and sets in only once the molecule has approached the surface to about $3 \AA$. We conclude that in the present system, the frozen-surface approximation is well justified for reflected molecules. If, however, a molecule has a high enough energy to overcome the barrier, it necessarily dissociates and the mobility of the surface $\mathrm{Al}$ atoms is then crucial in determining the further trajectory.

\section{DISCUSSION}

The results presented in the last section can be summarized in that the experimental low sticking probability for thermal molecules cannot be explained within an adiabatic picture. When confining the motion of the incoming molecules to the spin-triplet PES, there is some variation depending on the employed xc functional, but overall a significant lowering of the sticking coefficient is obtained. Of course, the latter represents only the opposite to the adiabatic picture: In the adiabatic picture, the electronic structure adapts instantaneously to the nuclear motion, and in the spintriplet motion, the electronic structure remains always in the initial gas-phase spin state. In reality, transitions away from this spin-triplet PES to other constrained states may occur. However, before embarking on this, it is necessary to assess, how well the employed xc functional may actually describe these various states, which we discuss here first for the isolated $\mathrm{O}_{2}$ molecule.

\section{A. $\mathrm{O}_{2}$ multiplets}

It is well known that in DFT the description of multiplets can be substantially incorrect. ${ }^{70}$ This is because the central quantity in DFT is the electron density, while the nodal structure of the wave function is not explicitly taken into account. The tiny differences in the electron (and magnetization) densities of nondegenerate multiplets are not resolved in present-day LDA or GGA xc functionals so that these multiplets turn out as degenerate. Similarly, degenerate multiplets that do differ in the electron (and magnetization) densities may be recognized as nondegenerate. This problem has been analyzed by Gunnarsson and Jones for a series of molecular states, ${ }^{58,70,76,77}$ showing that the origin of this limitation lies in the construction of the electron density from a 


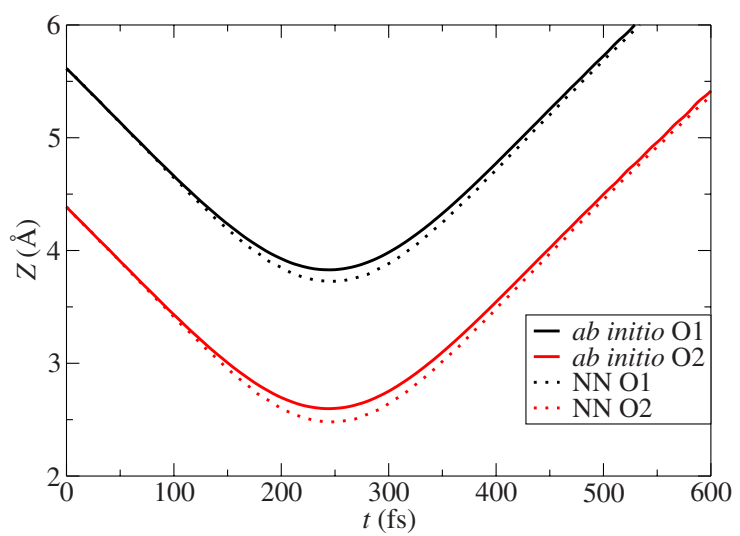

FIG. 9. (Color online) Comparison of a molecular trajectory on the spin-triplet PES obtained once with the NN-interpolated PES employing the frozen-surface approximation (solid lines) and once with a direct $a b$ initio $\mathrm{MD}$ run allowing full motion of the $\mathrm{Al}$ atoms (dotted lines). The molecule is initially located above the fcc site and oriented perpendicular to the surface with an initial kinetic energy of $0.15 \mathrm{eV}$. Shown is the vertical distance of the $\mathrm{O}$ atoms from the rigid first layer $\mathrm{Al}$ atoms as a function of time $t$. The atom closer to the surface is labeled with "O2," and the atom further away from the surface with "O1." Due to the onset of the energy barriers the turning point of the molecule is far away from the surface. No significant coupling to the motion of the surface atoms occurs and the NN and $a b$ initio trajectories are basically identical.

single determinant of Kohn-Sham orbitals. This typically yields an accurate energy with current GGA functionals if the wave function of the system can be represented correctly by a single determinant. If, however, the wave function is a combination of two or more determinants, then the symmetry information of the state is not taken into account in presentday xc functionals. ${ }^{77-79}$

The oxygen molecule is a diradical, i.e., the highest occupied molecular orbital, the antibonding $2 \pi^{*}$ orbital, is twofold degenerate, and occupied by two electrons. ${ }^{80}$ The lowlying singlet and triplet states of the $\mathrm{O}_{2}$ molecule, which differ only in the occupation of this orbital, are given by the following wave functions:

$$
\begin{gathered}
\psi_{1}^{s}=\frac{1}{2}\left[\phi_{1} \phi_{1}-\phi_{2} \phi_{2}\right](\alpha \beta-\beta \alpha), \\
\psi_{2}^{s}=\frac{1}{2}\left[\phi_{1} \phi_{1}+\phi_{2} \phi_{2}\right](\alpha \beta-\beta \alpha), \\
\psi_{3}^{s}=\frac{1}{2}\left[\phi_{1} \phi_{2}+\phi_{2} \phi_{1}\right](\alpha \beta-\beta \alpha), \\
\psi_{4}^{t}=\frac{1}{2}\left[\phi_{1} \phi_{2}-\phi_{2} \phi_{1}\right](\alpha \beta+\beta \alpha), \\
\psi_{5}^{t}=\frac{1}{\sqrt{2}}\left[\phi_{1} \phi_{2}-\phi_{2} \phi_{1}\right](\alpha \alpha),
\end{gathered}
$$
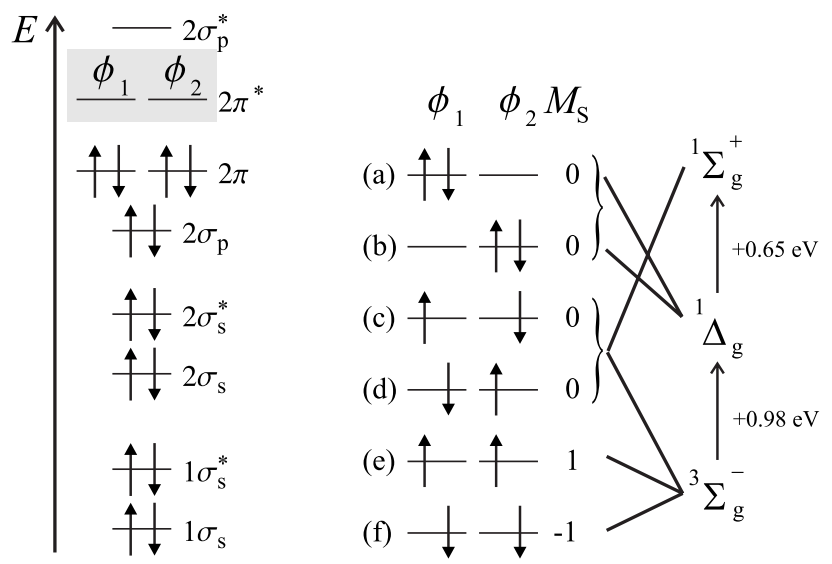

FIG. 10. Molecular orbital diagram of the $\mathrm{O}_{2}$ molecule. The ${ }^{3} \Sigma_{g}^{-}$ ground state and the two excited ${ }^{1} \Delta_{g}$ and ${ }^{1} \Sigma_{g}^{+}$singlet states differ only in the occupation numbers of the doubly degenerate $2 \pi^{*}$ state shown in gray on the left side. The six possible occupations are shown in (a)-(f) on the right side. Since open shell determinants are no eigenfunctions of the spin operator unless all electrons have parallel spin, four of the six oxygen wave functions are linear combinations of the determinants (a)-(d). Additionally shown are the measured energy gaps between the triplet and singlet states (Ref. $62)$.

$$
\psi_{6}^{t}=\frac{1}{\sqrt{2}}\left[\phi_{1} \phi_{2}-\phi_{2} \phi_{1}\right](\beta \beta) .
$$

Here, we have used for the involved spatial orbitals the short-hand notation $\phi_{1} \phi_{1}=\phi_{1}(1) \phi_{1}(2)$ and for the spin functions $\alpha \beta=\alpha(1) \beta(2)$, omitting the electronic labels. There are thus three degenerate triplet $(t)^{3} \Sigma_{g}^{-}$states $\left(\psi_{4}^{t}, \psi_{5}^{t}, \psi_{6}^{t}\right)$, a twofold degenerate singlet $(s){ }^{1} \Delta_{g}$ state $\left(\psi_{1}^{s}, \psi_{2}^{s}\right)$, and a higher singlet ${ }^{1} \Sigma_{g}^{+}$state $\left(\psi_{3}^{S}\right)$. The experimental singlet-triplet gap between the ${ }^{3} \Sigma_{g}^{-}$and the ${ }^{1} \Delta_{g}$ state for the free molecule is about $0.98 \mathrm{eV}$, and between the ${ }^{3} \Sigma_{g}^{-}$and the ${ }^{1} \Sigma_{g}^{+}$state the energy difference is $1.63 \mathrm{eV}{ }^{62}$ The energy diagram of the oxygen states is shown schematically in Fig. 10. The triplet states shown in Figs. 10(e) and 10(f) are represented by single determinants ("high-spin" triplets) and we may consequently expect that Kohn-Sham DFT with LDA or GGA functionals is in principle able to properly describe these triplet states. In a standard spin-polarized calculation, they are found as the ground state of $\mathrm{O}_{2}$, and correspondingly, we are confident that the calculated spin-triplet constrained PES correctly describes the initial state in the dissociation process at the $\mathrm{Al}(111)$ surface.

For the singlet states, which like the triplet state are diradicals, the situation is different. None of the singlet wave functions can be represented by a single determinant and it is thus a priori not clear if these states are described sufficiently accurately by Kohn-Sham DFT with the present-day $x c$ functionals. For the singlet-triplet gap, i.e., the energy difference by which the singlet state is higher than the triplet state, we compute half of the experimental value $(0.392 \mathrm{eV}$ for PBE and $0.393 \mathrm{eV}$ for RPBE). The reason is that a symmetry-broken electron density is obtained corresponding 
to one of the electronic configurations (c) and (d) in Fig. 10 with a nonzero magnetization density. This is a common observation in Kohn-Sham DFT. ${ }^{81,82}$ In the same way as $\psi_{3}^{s}$ and $\psi_{4}^{t}$ are linear combinations of the two Slater determinants (c) and (d) in Fig. 10, the single Slater determinant corresponding to the electronic configuration that we obtained is a combination of the "low-spin" triplet ${ }^{3} \Sigma_{g}^{-}$and the ${ }^{1} \Sigma_{g}^{+}$state, which is commonly referred to as "spin contamination." 83 Because of the large error in the computed singlet-triplet gap and the wrong spin-up and down densities, we conclude that Kohn-Sham DFT with current xc functionals does not allow us to calculate the singlet state with sufficient accuracy. An alternative without proper formal justification is to calculate a singlet state simply using non-spinpolarized DFT calculations. In this case, the magnetization density is zero everywhere in space. The calculations are straightforward and yield a singlet triplet gap of $1.138 \mathrm{eV}$ (PBE) and $1.171 \mathrm{eV}$ (RPBE) for the free $\mathrm{O}_{2}$ molecule, i.e., much closer to the experimental value.

Yet, another possibility to obtain the singlet-PES would be an application of the "sum method" of Ziegler et al. ${ }^{78} \mathrm{In}$ this method, the energy of the singlet state is calculated from the single-determinant singlet energy and the energy of the triplet state. This approach, as well as the nonspinpolarized approach, involves the mapping of a singlet PES and both are thus expected to lead to similar computational costs. For the free $\mathrm{O}_{2}$ molecule, also the results are essentially the same. Since the relevant part of the singlet-PES for the sticking coefficient is the one located at rather large distances from the surface, i.e., the part where the singlet-PES is still quite gas-phase-like, we therefore expect that the sum method and the approach based on non-spin-polarized calculations that will be employed here yield essentially the same results.

\section{B. Effect of electronic transitions on the sticking curve}

The barriers found on the spin-triplet PES are located at rather large distances from the surface. As discussed in Sec. II, the energetic order of the various constrained states is then still predominantly governed by the gas-phase $\mathrm{O}_{2}$ excitation spectrum. With the ionic states still very high in energy (and thereby ruling out the charge transfer picture suggested by Hellman et al. ${ }^{19}$ ), the only alternative constrained state in closer energetic vicinity to the spin-triplet state is thus the spin-singlet state, which will be the first alternative constrained state to become lower in energy than the spintriplet state at decreasing molecule-surface distances, as schematically shown in Fig. 1. Motivated by Wigner's spinselection rules and because the interaction time is not very long (cf., Fig. 9), we had disregarded such transitions to the spin-singlet state in the MD simulations confined to the spintriplet PES. We now set out to scrutinize this assumption.

With decreasing molecule-surface distance, the coupling to the solid surface increases and thereby the spin-selection rules become weakened. Crucial for the analysis of possible electronic transitions from the spin-triplet to the spin-singlet state is therefore to determine the location of the highdimensional crossing seam of the two corresponding PESs and to compare this position to the location of the energy barriers on the spin-triplet PES. If the crossing is at distances from the surface larger than the onset of the barriers on the spin-triplet PES, electronic transitions to the spin-singlet state are likely to modify the result obtained for the sticking coefficient in the spin-triplet only MD simulations. If the crossing is at distances closer to the surface than the onset of barriers, low energy molecules will be reflected by the barriers on the spin-triplet PES before ever approaching the surface close enough for electronic transitions to play a role. In this case, the result for the sticking curve obtained with the spin-triplet MD simulations will be quite accurate.

Central for such an analysis is thus the knowledge of both the spin-triplet and the spin-singlet PESs. Specifically, it is only the parts of these PESs at the larger distances from the surface where the triplet barriers are located that are of relevance for the here discussed sticking coefficient. As discussed above, there are unfortunately strong indications that present-day xc functionals may not be able to describe the spin-singlet PES with sufficient accuracy. Motivated by the closeness of the calculated singlet-triplet gap for the free $\mathrm{O}_{2}$ molecule to the experimental value, ${ }^{84}$ we therefore choose to use non-spin-polarized calculations to obtain a spin-singlet PES, even though there is no formal justification that this should indeed yield a good approximation to the real spinsinglet state. A weak rationalization for this choice may be that with the correct singlet-triplet splitting at large distances from the surface, the right energetic displacement between the two PESs should be provided. If then the approximate spin-singlet PES has roughly the same shape as the real spinsinglet PES, this should be good enough to track the approximate location of the crossing seam between the two PESs, which is at present our only interest.

In Fig. 11, the minimum energy path for dissociation on the spin-triplet PES is shown for two different molecular orientations. Along this minimum energy path, the energetics is additionally shown for the two xc functionals for the adiabatic, the LC-DFT spin-triplet, and the non-spin-polarized approximate spin-singlet case. It can clearly be seen that the crossing of the spin-triplet and the spin-singlet PES is for both molecular orientations closer to the surface than the onset of the energy barriers on the spin-triplet PES. A similar result is obtained for molecules impinging at several other sites over the surface. Low energy, thermal molecules are therefore most likely reflected by the energy barriers on the spin-triplet PES before they can reach distances to the surface, where electronic transitions would set in with a higher probability. For the lower kinetic energies, we therefore do not expect notable changes in the sticking curve due to electronic transitions compared to the result obtained with the spin-triplet confined MD simulations. Molecules with a higher kinetic energy, on the other hand, may reach the crossing seam of the two PESs well before arriving at the triplet barrier geometry. Electronic transitions may then occur with a higher probability. However, molecules with a high kinetic energy of about $1 \mathrm{eV}$ do even dissociate on the spin-triplet PES and thus possible electronic transitions will not be able to increase the sticking coefficient much further compared to the anyway close to $100 \%$ value obtained by the spin-triplet only MD simulations. At best, electronic transi- 

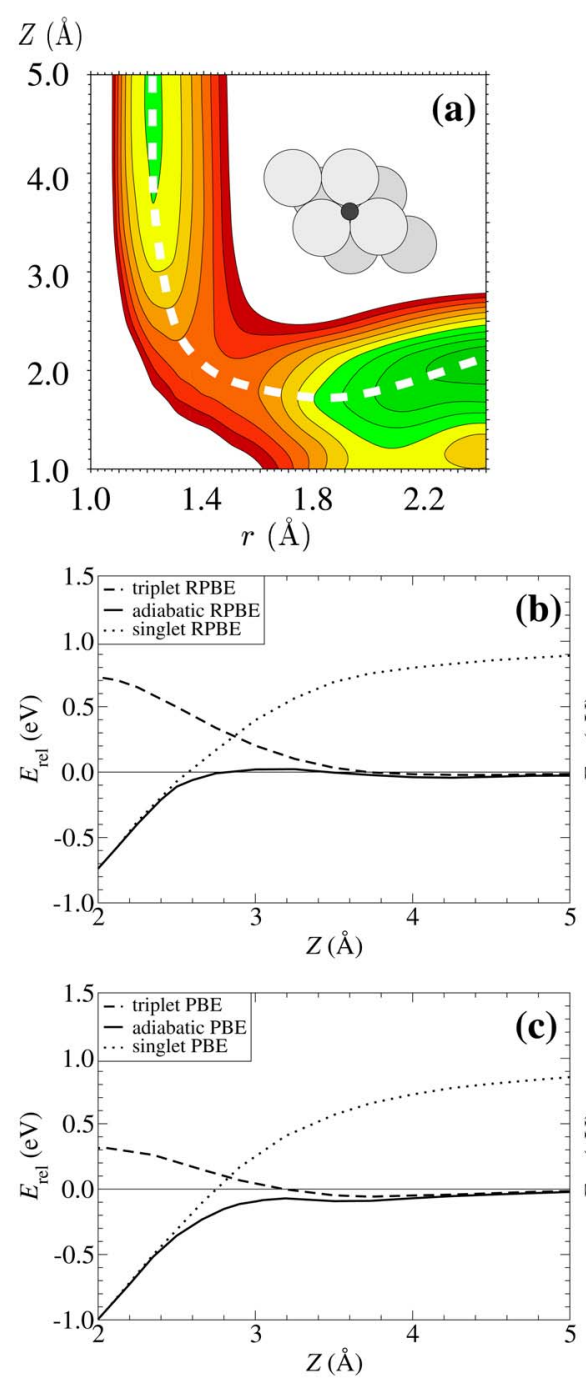
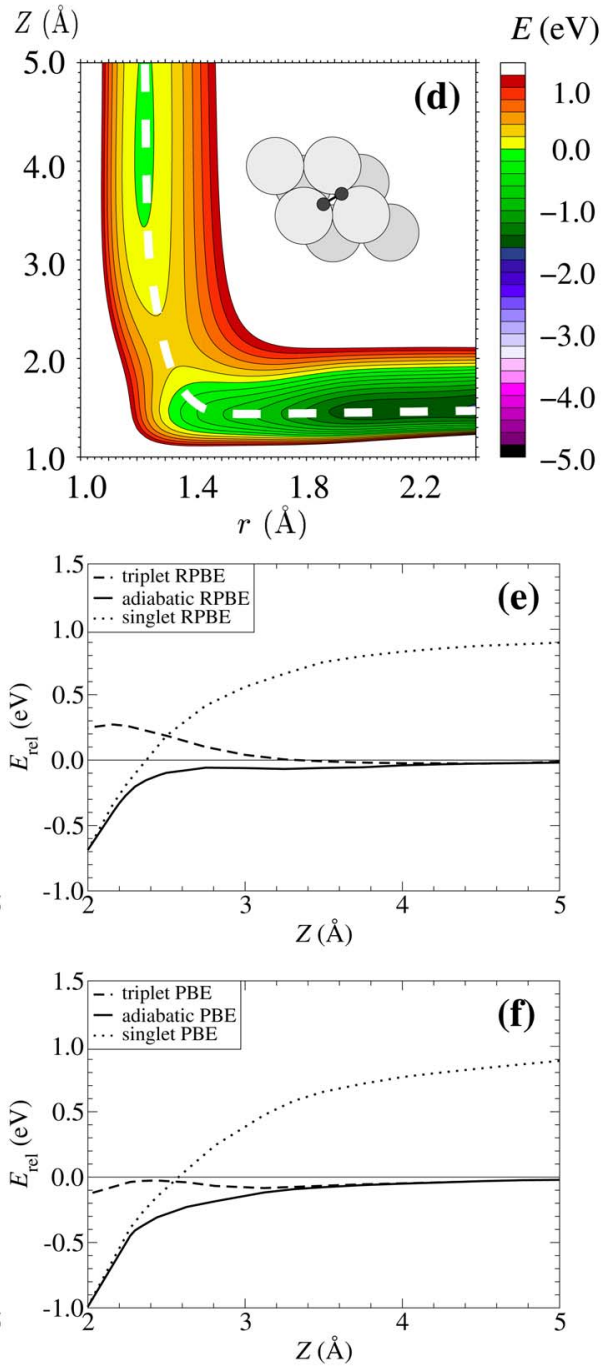

FIG. 11. (Color online) Energetics along the reaction path for a molecule impinging above the fcc site, with (a) a perpendicular and (d) a parallel orientation to the surface. The white dashed line corresponds to the minimum energy path for this molecular configuration. In (b) and (c), the energies of the adiabatic PES (black line), the approximate singlet PES (dotted line), and the LC-DFT spin-triplet PES (dashed line) along this path are shown as a function of the center-of-mass distance from the surface $Z$. (b) corresponds to the RPBE functional and (c) refers to the PBE functional. The corresponding PESs for the parallel orientation are shown in (e) for the RPBE and in (f) for the PBE functional. tions may therefore only have a notable effect on the sticking curve at intermediate kinetic energies, most likely further increasing the sticking probability. In this respect, it is intriguing to note that the spin-triplet confined sticking coefficient computed with the RPBE functional in Fig. 6 does indeed lie below the experimental curve at these intermediate energies. However, in light of the xc functional dependence discussed in Sec. IV B 2, it would be unwarranted to ascribe this difference directly to the effect of electronic transitions.

\section{CONCLUSION}

In the present paper, we have further detailed our firstprinciples investigation of the initial dissociative sticking of oxygen molecules at the $\mathrm{Al}(111)$ surface. We provide evidence that fundamental and undisputable experimental results like the low sticking probability for thermal oxygen molecules cannot be explained in terms of an adiabatic dissociation process. In order to show this, we calculated the adiabatic potential-energy surface in six-dimensions using density-functional theory. Subsequent interpolation by a neural network fitting procedure and extensive molecular dynamics simulations on the interpolated PES shows unambiguously that the adiabatic description yields a unit sticking probability independent of the molecular kinetic energy, in clear disagreement with the experimental data.

As most likely nonadiabatic effects cause this discrepancy, we focused on a hindered spin flip of the incoming $\mathrm{O}_{2}$ molecule away from the initial spin-triplet gas-phase state. For this purpose, we employed a locally constrained DFT method that allows us to localize the triplet spin at the oxygen molecule and were thereby able to compute the corresponding spin-triplet PES seen by such a molecule when impinging on the surface. In contrast to the adiabatic PES, this PES exhibits barriers in the molecular entrance channel. Consequently, we find a reduced sticking probability when confining the molecular motion to this spin-triplet PES. While we can argue that electronic transitions away from this PES to other states will have basically no effect on thermal molecules and will only slightly increase the sticking probability for higher kinetic energies, we do observe a substantial variation in the calculated sticking probability depending on the employed xc functional. Future studies employing a higher-level energetics are therefore desirable for a final quantitative comparison to the experimental data. 


\section{ACKNOWLEDGMENTS}

The authors gratefully acknowledge Bernard Delley for providing the $\mathrm{DMOL}^{3}$ code, into which we implemented our
LC-DFT approach. We wish to thank Eckart Hasselbrink and Bengt Lundqvist for stimulating discussions and gratefully acknowledge partial funding from the Deutsche Forschungsgemeinschaft.
*Present address: Lehrstuhl für Theoretische Chemie, RuhrUniversität Bochum, D-44780 Bochum, Germany.

${ }^{1}$ H. Brune, J. Wintterlin, R. J. Behm, and G. Ertl, Phys. Rev. Lett. 68, 624 (1992).

${ }^{2}$ H. Brune, J. Wintterlin, J. Trost, G. Ertl, J. Wiechers, and R. J. Behm, J. Chem. Phys. 99, 2128 (1993).

${ }^{3}$ M. Binetti, O. Weisse, E. Hasselbrink, A. J. Komrowski, and A. C. Kummel, Faraday Discuss. 117, 313 (2000).

${ }^{4}$ A. J. Komrowski, J. Z. Sexton, A. C. Kummel, M. Binetti, O. Weisse, and E. Hasselbrink, Phys. Rev. Lett. 87, 246103 (2001).

${ }^{5}$ M. Schmid, G. Leonardelli, R. Tscheliessnig, A. Biedermann, and P. Varga, Surf. Sci. Lett. 478, L355 (2001).

${ }^{6}$ P. O. Gartland, Surf. Sci. 62, 183 (1977).

${ }^{7}$ L. Österlund, I. Zorić, and B. Kasemo, Phys. Rev. B 55, 15452 (1997).

${ }^{8}$ A. M. Bradshaw, P. Hofmann, and W. Wyrobisch, Surf. Sci. 68, 269 (1977)

${ }^{9}$ V. Zhukov, I. Popova, and J. T. Yates, Jr., J. Vac. Sci. Technol. A 17, 1727 (1999)

${ }^{10}$ C. S. Lee and T. M. Lin, Surf. Sci. 471, 219 (2001).

${ }^{11}$ K. Honkala and K. Laasonen, Phys. Rev. Lett. 84, 705 (2000).

${ }^{12}$ Y. Yourdshahyan, B. Razaznejad, and B. I. Lundqvist, Solid State Commun. 117, 531 (2001).

${ }^{13}$ Y. Yourdshahyan, B. Razaznejad, and B. I. Lundqvist, Phys. Rev. B 65, 075416 (2002).

${ }^{14}$ B. Kasemo, Phys. Rev. Lett. 32, 1114 (1974).

${ }^{15}$ B. Kasemo, R. Toernqvist, J. K. Nørskov, and B. I. Lundqvist, Surf. Sci. 89, 554 (1979).

${ }^{16}$ G. Katz, Y. Zeiri, and R. Kosloff, J. Chem. Phys. 120, 3931 (2004).

${ }^{17}$ A. M. Wodtke, J. C. Tully, and D. J. Auerbach, Int. Rev. Phys. Chem. 23, 513 (2004).

${ }^{18}$ A. Hellman, B. Razaznejad, Y. Yourdshahyan, H. Ternow, I. Zorić, and B. I. Lundqvist, Surf. Sci. 532-535, 126 (2003).

${ }^{19}$ A. Hellman, B. Razaznejad, and B. I. Lundqvist, Phys. Rev. B 71, 205424 (2005).

${ }^{20}$ A. Böttcher, R. Imbeck, A. Morgante, and G. Ertl, Phys. Rev. Lett. 65, 2035 (1990).

${ }^{21}$ T. Greber, Chem. Phys. Lett. 222, 292 (1994).

${ }^{22}$ V. P. Zhdanov, Phys. Rev. B 55, 6770 (1997).

${ }^{23}$ K. Kato and T. Uda, Phys. Rev. B 62, 15978 (2000).

${ }^{24}$ K. Kato, T. Uda, and K. Terakura, Phys. Rev. Lett. 80, 2000 (1998).

${ }^{25}$ J. Behler, B. Delley, S. Lorenz, K. Reuter, and M. Scheffler, Phys. Rev. Lett. 94, 036104 (2005).

${ }^{26}$ E. Wigner, Nachr. Ges. Wiss. Goettingen, Math.-Phys. K1. 375 (1927).

${ }^{27}$ J. Behler, B. Delley, K. Reuter, and M. Scheffler, Phys. Rev. B 75, 115409 (2007).

${ }^{28}$ M. Born and R. Oppenheimer, Ann. Phys. 84, 457 (1927).

${ }^{29}$ G. J. Kroes, E. J. Baerends, and R. C. Mowrey, Phys. Rev. Lett.
78, 3583 (1997).

${ }^{30}$ P. Nieto, E. Pijper, D. Barredo, G. Laurent, R. A. Olsen, E. J. Baerends, G. J. Kroes, and D. Farias, Science 312, 86 (2006).

${ }^{31}$ A. Gross, S. Wilke, and M. Scheffler, Phys. Rev. Lett. 75, 2718 (1995).

${ }^{32}$ A. Gross and M. Scheffler, Phys. Rev. B 57, 2493 (1998).

${ }^{33}$ G. Kresse, Phys. Rev. B 62, 8295 (2000).

${ }^{34}$ G. Wiesenekker, G. J. Kroes, and E. J. Baerends, J. Chem. Phys. 104, 7344 (1996).

${ }^{35}$ J. Dai and J. Light, J. Chem. Phys. 107, 1676 (1997).

${ }^{36}$ C. M. Wei, A. Gross, and M. Scheffler, Phys. Rev. B 57, 15572 (1998).

${ }^{37}$ T. Klüner, H.-J. Freund, V. Staemmler, and R. Kosloff, Phys. Rev. Lett. 80, 5208 (1998).

${ }^{38}$ A. Gross, M. Scheffler, M. J. Mehl, and D. A. Papaconstantopoulos, Phys. Rev. Lett. 82, 1209 (1999).

${ }^{39}$ A. Gross, A. Eichler, J. Hafner, M. J. Mehl, and D. A. Papaconstantopoulos, Surf. Sci. 539, L542 (2003).

${ }^{40}$ M. J. Mehl and D. A. Papaconstantopoulos, Phys. Rev. B 54, 4519 (1996).

${ }^{41}$ R. E. Cohen, M. J. Mehl, and D. A. Papaconstantopoulos, Phys. Rev. B 50, 14694 (1994).

${ }^{42}$ D. E. Makarov and H. Metiu, J. Chem. Phys. 108, 590 (1998).

${ }^{43}$ R. P. A. Bettens and M. A. Collins, J. Chem. Phys. 111, 816 (1999).

${ }^{44}$ C. Crespos, M. A. Collins, E. Pijper, and G. J. Kroes, Chem. Phys. Lett. 376, 566 (2003).

${ }^{45}$ H. F. Busnengo, W. Dong, and A. Salin, Chem. Phys. Lett. 320, 328 (2000).

${ }^{46}$ T. B. Blank, S. D. Brown, A. W. Calhoun, and D. J. Doren, J. Chem. Phys. 103, 4129 (1995).

${ }^{47}$ D. F. R. Brown, M. N. Gibbs, and D. C. Clary, J. Chem. Phys. 105, 7597 (1996).

${ }^{48}$ S. Lorenz, A. Gross, and M. Scheffler, Chem. Phys. Lett. 395, 210 (2004).

${ }^{49}$ S. Lorenz, M. Scheffler, and A. Gross, Phys. Rev. B 73, 115431 (2006).

${ }^{50} \mathrm{DMOL}^{3}$, academic version [B. Delley, J. Chem. Phys. 92, 508 (1990)].

${ }^{51}$ B. Delley, J. Chem. Phys. 113, 7756 (2000).

${ }^{52}$ P. Pulay, Chem. Phys. Lett. 73, 393 (1980).

${ }^{53}$ P. Pulay, J. Comput. Chem. 3, 556 (1982).

${ }^{54}$ F. Wagner, T. Laloyaux, and M. Scheffler, Phys. Rev. B 57, 2102 (1998).

${ }^{55}$ J. P. Perdew, K. Burke, and M. Ernzerhof, Phys. Rev. Lett. 77, 3865 (1996).

${ }^{56}$ B. Hammer, L. B. Hansen, and J. K. Nørskov, Phys. Rev. B 59, 7413 (1999).

${ }^{57}$ J. P. Perdew, J. A. Chevary, S. H. Vosko, K. A. Jackson, M. R. Pederson, D. J. Singh, and C. Fiolhais, Phys. Rev. B 46, 6671 (1992). 
${ }^{58}$ O. Gunnarsson and R. O. Jones, Phys. Rev. B 31, 7588 (1985).

${ }^{59}$ J. P. Perdew, J. A. Chevary, S. H. Vosko, K. A. Jackson, M. R. Pederson, D. J. Singh, and C. Fiolhais, Phys. Rev. B 46, 6671 (1992).

${ }^{60}$ P. M. W. Gill, B. G. Johnson, J. A. Pople, and M. J. Frisch, Int. J. Quantum Chem. 26, 319 (1992).

${ }^{61}$ G. Herzberg, Can. J. Phys. 30, 185 (1952).

${ }^{62}$ G. Herzberg, Molecular Spectra and Molecular Structure. I. Spectra of Diatomic Molecules (van Nostrand Reinhold, New York 1950).

${ }^{63}$ J. Jacobsen, B. Hammer, K. W. Jacobsen, and J. K. Nørskov, Phys. Rev. B 52, 14954 (1995).

${ }^{64}$ C. J. Fall, N. Binggeli, and A. Baldereschi, Phys. Rev. B 58, R7544 (1998).

${ }^{65}$ A. Kiejna and B. I. Lundqvist, Phys. Rev. B 63, 085405 (2001).

${ }^{66}$ A. Gross, Surf. Sci. Rep. 32, 291 (1998).

${ }^{67}$ P. H. Dederichs, S. Blügel, R. Zeller, and H. Akai, Phys. Rev. Lett. 53, 2512 (1984).

${ }^{68}$ Q. Wu and T. Van Voorhis, Phys. Rev. A 72, 024502 (2005).

${ }^{69}$ P. Hohenberg and W. Kohn, Phys. Rev. 136, B864 (1964).

${ }^{70}$ R. O. Jones and O. Gunnarsson, Rev. Mod. Phys. 61, 689 (1989).

${ }^{71}$ J. Behler, S. Lorenz, and K. Reuter, J. Chem. Phys. 127, 014705 (2007).

${ }^{72}$ K. Hornik, Neural Networks 2, 359 (1989).

${ }^{73}$ W. H. Press, S. A. Teukolsky, W. T. Vetterling, and B. P. Flannery, Numerical Recipes in Fortran 77 (Cambridge University Press, Cambridge, 1992).

${ }^{74}$ K. Schwarz and P. Mohn, J. Phys. F: Met. Phys. 14, L129 (1984).

${ }^{75}$ In the case of the spin-triplet PES, only the PBE energies have been calculated fully self-consistently. Starting from the selfconsistent PBE energies, the RPBE energies have been obtained from one additional iteration employing this functional. We have carefully checked that the error introduced by this non-selfconsistent procedure is negligible by comparing the results of 96 fully self-consistent RPBE energies with the corresponding nonself-consistent values. The average error in energy differences between different molecular configurations was found to be about $1.3 \mathrm{meV}$, while the maximum error found was $6 \mathrm{meV}$.

${ }^{76}$ O. Gunnarsson, J. Harris, and R. O. Jones, J. Chem. Phys. 67, 3970 (1977).

${ }^{77}$ O. Gunnarsson and R. O. Jones, J. Chem. Phys. 72, 5357 (1980).

${ }^{78}$ T. Ziegler, A. Rauk, and E. J. Baerends, Theor. Chim. Acta 43, 261 (1977).

${ }^{79}$ U. von Barth, Phys. Rev. A 20, 1693 (1979).

${ }^{80}$ L. Salem and C. Rowland, Angew. Chem., Int. Ed. Engl. 11, 92 (1972).

${ }^{81}$ A. A. Ovchinnikov and J. K. Labanowski, Phys. Rev. A 53, 3946 (1996).

${ }^{82}$ D. C. Sorescu, K. D. Jordan, and P. Avouris, J. Phys. Chem. B 105, 11227 (2001).

${ }^{83}$ W. J. Hehre, L. Radom, P. V. R. Schleyer, and J. A. Pople, $A b$ initio Molecular Orbital Theory (Wiley, New York, 1986).

${ }^{84}$ When calculating the energetic difference between the spin-triplet PES and the approximate spin-singlet PES obtained by the nonspin-polarized calculations, we obtain 0.898 and $0.937 \mathrm{eV}$ for the PBE and RPBE xc functional at large molecule-surface distances $(6 \AA)$, respectively. These values differ from those obtained for the isolated $\mathrm{O}_{2}$ molecule by about $0.2 \mathrm{eV}$. The reason is again the uncontrolled charge transfer to the oxygen $2 \pi^{*}$ orbital that has been discussed in detail in Ref. 27. This charge transfer artificially lowers the total energy of the non-spinpolarized singlet PES by coincidence such that almost exactly the experimental value is obtained for the singlet-triplet gap. 